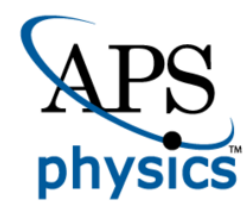

This is the accepted manuscript made available via CHORUS. The article has been published as:

\title{
Anomalous spin precession and spin Hall effect in semiconductor quantum wells
}

Xintao Bi, Peiru He, E. M. Hankiewicz, R. Winkler, Giovanni Vignale, and Dimitrie Culcer Phys. Rev. B 88, 035316 - Published 29 July 2013 DOI: 10.1103/PhysRevB.88.035316 


\title{
Anomalous spin precession and spin Hall effect in semiconductor quantum wells
}

\author{
Xintao Bi,${ }^{1}$ Peiru He, ${ }^{1}$ E. M. Hankiewicz, ${ }^{2}$ R. Winkler, ${ }^{3,4}$ Giovanni Vignale, ${ }^{5}$ and Dimitrie Culcer ${ }^{1,6}$ \\ ${ }^{1}$ ICQD, Hefei National Laboratory for Physical Sciences at the Microscale, \\ University of Science and Technology of China, Hefei, Anhui 230026, China \\ ${ }^{2}$ Institut für Theoretische Physik und Astrophysik, \\ Universität Würzburg, 97074 Würzburg, Germany \\ ${ }^{3}$ Materials Science Division, Argonne National Laboratory, Argonne, IL 60439. \\ ${ }^{4}$ Northern Illinois University, De Kalb, IL 60115. \\ ${ }^{5}$ Department of Physics and Astronomy, University of Missouri, Columbia, Missouri 65211 \\ ${ }^{6}$ School of Physics, The University of New South Wales, Sydney 2052, Australia
}

(Dated: June 6, 2013)

\begin{abstract}
SO (SO) interactions give a spin-dependent correction $\hat{\boldsymbol{r}}_{s o}$ to the position operator, referred to as the anomalous position operator. We study the contributions of $\hat{\boldsymbol{r}}_{\text {so }}$ to the spin-Hall effect (SHE) in quasi two-dimensional (2D) semiconductor quantum wells with strong band structure SO interactions that cause spin precession. The skew scattering and side-jump scattering terms in the SHE vanish, but we identify two additional terms in the SHE, due to $\hat{\boldsymbol{r}}_{s o}$, which have not been considered in the literature so far. One term reflects the modification of spin precession due to the action of the external electric field (the field drives the current in the quantum well), which produces, via $\hat{\boldsymbol{r}}_{s o}$, an effective magnetic field perpendicular to the plane of the quantum well. The other term reflects a similar modification of spin precession due to the action of the electric field created by random impurities, and appears in a careful formulation of the Born approximation. We refer to these two effects collectively as anomalous spin precession and we note that they contribute to the SHE to the first order in the SO coupling constant even though they formally appear to be of second order. In electron systems with weak momentum scattering, the contribution of the anomalous spin precession due to the external electric field equals $1 / 2$ the usual side-jump SHE, while the additional impurity-dependent contribution depends on the form of the band structure SO coupling. For band structure SO coupling linear in wave vector the two anomalous spin precession contributions cancel. For band structure SO coupling cubic in wave vector, however, they do not cancel, and the anomalous spin precession contribution to the SHE can be detected in a high-mobility 2DEG with strong SO coupling. In 2D hole systems both anomalous spin precession contributions vanish identically.
\end{abstract}

PACS numbers:

\section{INTRODUCTION}

In systems with strong spin-orbit (SO) interactions an electric field generates a transverse spin-current ${ }^{1-12}$ : this phenomenon is referred to as the spin-Hall effect (SHE). For the past ten years, the SHE has been a source of new ideas for magneto-electronic devices ${ }^{13}$ aimed at integrating semiconductor and magnetic technologies, facilitating efficient information processing and quantum computing architectures. ${ }^{14-17}$ These visions have stimulated a large volume of experimental and theoretical work. ${ }^{18-33}$ Experimentally, the SHE was initially studied in semiconductors, ${ }^{34-38}$ but has since expanded to novel materials such as HgTe-based quantum wells, ${ }^{39}$ topological insulators and graphene, and $d$-band metals. ${ }^{40-42}$ It is often simpler to measure the inverse spin-Hall effect, ${ }^{43}$ where a spin current generates a transverse charge current, which is detected by conventional means. The inverse SHE has been observed in $\mathrm{Al},{ }^{44} \mathrm{Pt}$ wires at room temperature, ${ }^{45}$ hybrid FePt/Au devices, ${ }^{46}$ $\mathrm{Au}$ films with $\mathrm{Pt}$ impurities, ${ }^{47}$ permalloy/normal metal bilayers, ${ }^{48}$ GaAs multiple quantum wells, ${ }^{49}$ and $\mathrm{Cu}$ with Ir impurities. ${ }^{50}$ For a review of recent experimental work on the SHE in Pt see Ref. 51. Observation of the in- verse SHE has recently been reported even in a weakly $\mathrm{SO}$ coupled material such as $\mathrm{Si}^{52}$

SO coupling may be present in the band structure and in the impurity potentials. Band-structure SO interactions become important in structures lacking a center of inversion when SO interactions lift spin degeneracy. ${ }^{77}$ If the underlying crystal lattice lacks a center of inversion the material is said to possess bulk inversion asymmetry (BIA). In low-dimensionsional systems the confinement potential can be made asymmetric, in which case one speaks of structure inversion asymmetry (SIA). In this paper we consider exclusively quasi two-dimensional semiconductor systems that lack a center of inversion due to $\mathrm{BIA}^{78}$ and/or SIA giving rise to Rashba SO coupling. In these systems the band structure SO interaction is represented by a Hamiltonian $H=(\hbar / 2) \boldsymbol{\sigma} \cdot \boldsymbol{\Omega}_{\boldsymbol{k}}$ describing the interaction of the spin with an effective wave vector-dependent magnetic field $\boldsymbol{\Omega}_{\boldsymbol{k}}$. This can be $\boldsymbol{\Omega}_{k}^{\mathrm{BIA}}$ or $\boldsymbol{\Omega}_{k}^{\mathrm{SIA}}$. The spin precesses about this field with frequency $\Omega_{k} \equiv\left|\Omega_{k}\right|$. Different physical regimes are distinguished by the value of the product of $\Omega_{k}$ with the momentum relaxation time $\tau_{p}$. In the ballistic regime (clean limit) $\Omega_{\boldsymbol{k}} \tau_{p} \rightarrow \infty$. The weak momentum scattering regime is characterized by $\Omega_{\boldsymbol{k}} \tau_{p} \gg 1$, while in the 
strong momentum scattering regime $\Omega_{\boldsymbol{k}} \tau_{p} \ll 1$.

SO interactions arise, quite generally, from a spindependent correction $\hat{\boldsymbol{r}}_{s o}$ to the position operator, ${ }^{53,54}$ whose general form is

$$
\hat{\boldsymbol{r}}_{s o}=\lambda \boldsymbol{\sigma} \times \boldsymbol{\omega}_{\boldsymbol{k}},
$$

where $\lambda$ is a material-specific parameter, $\boldsymbol{\sigma}$ is the vector of Pauli spin matrices, and $\boldsymbol{\omega}_{\boldsymbol{k}}$ takes different forms for different systems, as well as for electrons and holes in the same system. ${ }^{79}$ We note that $\boldsymbol{\Omega}_{\boldsymbol{k}}$ and $\boldsymbol{\omega}_{\boldsymbol{k}}$ are not independent of each other. The $\boldsymbol{\omega}_{\boldsymbol{k}}$ entering the corrected position operator is inherently related to $\boldsymbol{\Omega}_{\boldsymbol{k}}^{\text {SIA }}$ characterizing the Rashba SO coupling through a term of the form $\boldsymbol{\Omega}_{k}^{\text {SIA }}=\lambda \boldsymbol{\omega}_{\boldsymbol{k}} \times \nabla V$. The presence of $\hat{\boldsymbol{r}}_{s o}$ results in corrections to the interaction between charge carriers and electric fields, which include impurities and external electric fields. Thus, in addition to the band structure SO interaction, one must take into account SO interactions arising from the external electric field and from the electron-impurity potential. The interplay between these interactions in the SHE is quite a complicated subject. It has received a lot of attention in recent years, yet, as we will see, it is not yet completely understood.

Perhaps the most intuitive mechanism of SHE is the one known as skew scattering, i.e., the asymmetric scattering of up and down spins by impurities. ${ }^{55-57}$ Next, we have the so-called side-jump scattering term, ${ }^{53,58-60}$ which consists of two equal terms, one reflecting the correction to the band energy due to the spin-dependent interaction with the electric field, the other reflecting the renormalization of the carrier trajectory during collisions. Diagrammatic formulations naturally recover the two side-jump scattering terms through the vertex renormalization of spin and charge currents, as Ref. 56 demonstrated. An analytical derivation of the side jump from the Kubo formula was presented in Ref. 61. Furthermore, Ref. 10 identified skew-scattering and side-jump scattering within a drift-diffusion approach. More recently, side jump scattering was derived starting from the quantum Liouville equation for the single-particle spin density-matrix. ${ }^{62}$

The analysis of the SHE becomes considerably more complicated when both band-structure and impuritypotential induced SO interactions are present. This problem was first addressed by Tse and Das Sarma, ${ }^{63}$ who employed the diagrammatic Kubo formula and considered band structure SO coupling of the linear Rashba form. They found that the skew scattering contribution to the SHE vanished for arbitrarily small value of the band structure SO coupling, while a term equal to half the usual side-jump scattering SHE survived ${ }^{80}$ This is in contrast to the result obtained in Ref. 64 that both the side-jump and the skew scattering contributions vanish for arbitrarily small values of the band structure SO coupling, as long as impurity-induced (Elliot-Yafet) spin relaxation is neglected. These two results are reconciled by taking into account the SO contribution to the electronimpurity self-energy diagram, ${ }^{65}$ which recovers the van- ishing of the side-jump and skew scattering contributions found in Ref. 64.

The principal question identified in Ref. 63 was the paradox of the non-analyticity of the spin Hall conductivity, which appears to change discontinuously as soon as the band structure SO coupling is turned on. This paradox was finally solved in Ref. 66 by the introduction of an impurity-induced (Elliott-Yafet) spin relaxation rate $1 / \tau_{\mathrm{EY}}$, which led to a spin Hall conductivity of the form

$$
\sigma_{y x}^{z}=\frac{\left[\sigma_{y x}^{z}\right]_{s s}+\left[\sigma_{y x}^{z}\right]_{s j}}{1+\tau_{\mathrm{EY}} / \tau_{\mathrm{DP}}}
$$

where $\tau_{\mathrm{DP}}$ is the Dyakonov-Perel relaxation time associated with the band structure SO coupling and given by $\tau_{\mathrm{DP}}^{-1}=\left\langle\Omega_{k}^{2}\right\rangle \tau_{p}$, where $\tau_{p}$ is the momentum relaxation time and the angular bracket denotes an average over the momentum distribution. The above formula exhibits a smooth crossover between the sum of skew-scattering (ss) and side-jump scattering (sj) contributions, when the band structure spin precession $\Omega_{k}$ is neglected, and zero when $\Omega_{k} \tau_{p} \gg 1$, i.e. when the band-structure SO interaction is much stronger than the electron-impurity interaction (see also Ref. 65).

However, this is not the end of the story. The work described above was limited to band-structure SO couplings that are linear in wave vector $\boldsymbol{k}$. The aim of this work is to provide a consistent framework for treating band structure and impurity SO effects in quasi twodimensional quantum wells for any form of the band structure $S O$ interaction in the weak momentum scattering regime $\Omega_{\boldsymbol{k}} \tau_{p} \gg 1$. To this end, we construct a rigorous theory of the interplay of spin precession due to band structure SO coupling and SO coupling due to impurities. We start from the quantum Liouville equation and derive a kinetic equation for the spin density matrix, which captures the effects of band-structure spin precession and $\hat{\boldsymbol{r}}_{s o}$ on an equal footing. We focus from the very beginning on the weak momentum scattering regime $\Omega_{\boldsymbol{k}} \tau_{p} \gg 1$. Under this assumption, we do not have to worry about the finite Elliot-Yafet scattering rate that appears in Eq. (2): we are in the regime $\tau_{\mathrm{EY}} \gg \tau_{\mathrm{DP}}$. But, while Eq. (2) predicts, in this limit, a vanishing spin Hall conductivity for linear-in- $\boldsymbol{k}$ band-structure SO interaction, we will show that a finite spin Hall conductivity can survive for different forms of that SO interaction.

More precisely, we find that, in the weak momentum scattering regime, skew scattering and side jump scattering still give zero SHE. At the same time, we identify two additional contributions to the SHE stemming from $\hat{\boldsymbol{r}}_{\text {so. }}$. These contributions have been overlooked in the literature thus far. One contribution arises from the impurity potential, and is found in the Born approximation when scattering terms of second order in $\mathrm{SO}$ are taken into account. This contribution can be viewed as a modification of the band structure precession frequency due to the electron-impurity interaction. The second contribution is scattering-independent. Its origin lies in the spin-dependent interaction with the external electric field 
brought about by $\hat{\boldsymbol{r}}_{s o}$. This has the form of an interaction between each carrier and an effective magnetic field. The carrier spin precesses in this effective magnetic field in such a way that an out-of-plane spin component is generated, which contributes to the SHE. We refer to these two effects collectively as anomalous spin precession. The impurity-induced anomalous spin precession term gives an out-of plane component of the effective magnetic field. This is precisely what distinguishes anomalous spin precession from the usual side-jump scattering term, which vanishes in the presence of spin-precession. Remarkably, these effects contribute to the SHE in the first order in the SO coupling constant even though they formally appear to be of second order. The external electric field part of the anomalous spin precession term appears to be universal in electronic systems in the clean limit.

In electron systems with band structure SO linear in $\boldsymbol{k}$ the sum of the two anomalous spin precession terms vanishes. In hole systems both additional terms are zero independently. Nevertheless, the anomalous spin precession term in the SHE in general survives, and we demonstrate its existence explicitly in 2D electron systems with band structure SO described by the cubic Dresselhaus model. In this model we find the total SHE conductivity in the clean limit to be [see Eq. (55) below]

$$
\sigma_{y x}^{z} \approx-\frac{e}{16 \pi}+\frac{n_{e} e \lambda}{4} .
$$

The term $\propto \lambda$ is linear in the electron number density, while the band-structure SO contribution in the weak momentum scattering regime is density-independent. The cubic Dresselhaus SO interaction term is strong in a wide electron quantum well at high density $n_{e}$. Although the experimental situation is more complicated than the above formula suggests (see Sec. VIII), and involves the non-trivial interplay of linear and cubic SO terms, we find that in a high-mobility 2D electron gas based on InSb, anomalous spin precession accounts for most of the spinHall conductivity. Our results are therefore relevant to experiments and help to distinguish different contributions to the SHE.

Contributions to the SHE purely from band structure SO are well known. ${ }^{6}$ We do not discuss them explicitly here, except in the practical case of experimental observation (Sec. VIII). The focus of this work is on the contributions to the SHE due to $\hat{\boldsymbol{r}}_{\text {so }}$, and the central result is that, aside from the well-known skew scattering and side-jump scattering terms, two additional contributions - the anomalous spin precession terms - are present when band structure SO is nonzero. To our knowledge, this is the first work that proves that $\hat{\boldsymbol{r}}_{\text {so }}$ can give rise to a spin-Hall current through a mechanism unrelated to scattering. We work up to third order in the impurity potential, and, in order to recover all contributions, we consider terms of second order in the SO coupling. Our results are valid in the weak momentum scattering limit, yet in the Appendix we prove rigorously that a non-analyticity in the strong momentum scattering limit is cured by introducing the Elliott-Yafet spin relaxation time $\tau_{\mathrm{EY}}$, as was done in Ref. 65 .

The outline of this paper is as follows. In Sec. II we present the band Hamiltonian and in Sec. III we discuss the effective position operator. In Sec. IV we derive the general form of the kinetic equation starting with the quantum Liouville equation, and discuss the various scattering terms. In Sec. V we discuss the non-equilibrium correction to the density matrix, demonstrating that a new, scattering-independent driving term due to $\hat{\boldsymbol{r}}_{\text {so }}$ is present. The general solution to the kinetic equation is presented in Sec. VI, demonstrating that the skew scattering and side-jump scattering terms give zero contributions to the SHE. All SHE contributions due to $\hat{\boldsymbol{r}}_{s o}$ are listed for commonly employed models of SO coupling. An explanation of anomalous spin precession is given in Sec. VII, which is followed by a detailed discussion of the experimental situation in Sec. VIII, and the summary and conclusions.

\section{BAND HAMILTONIAN}

In the crystal-momentum representation, the band Hamiltonian $\hat{H}_{0}$ in the effective mass approximation has the general form

$$
H_{0 \boldsymbol{k}}=H_{\mathrm{kin}}+H_{s o} \equiv H_{\mathrm{kin}}+\frac{\hbar}{2} \boldsymbol{\sigma} \cdot \boldsymbol{\Omega}_{\boldsymbol{k}},
$$

for an arbitrary SO interaction. The kinetic energy term $H_{\text {kin }}=\varepsilon_{0 \boldsymbol{k}} \mathbb{1} \equiv \frac{\hbar^{2} k^{2}}{2 m^{*}} \mathbb{1}$, where $\mathbb{1}$ is the identity matrix in spin space and $m^{*}$ the carrier effective mass. The spin-dependent term in the Hamiltonian $H_{s o}$ is treated as a perturbation with respect to the kinetic energy term. The eigen-energies are written as $\varepsilon_{\boldsymbol{k} \pm}=\varepsilon_{0 \boldsymbol{k}} \pm\left(\hbar \Omega_{\boldsymbol{k}} / 2\right)$.

For quasi-2D systems we may have different contributions to SO coupling that are relevant in different regimes. ${ }^{67}$ For $2 \mathrm{D}$ spin- $1 / 2$ electron systems with SIA, the band structure contains the linear Rashba Hamiltonian

$$
H_{R 1}=\alpha_{1}\left(\sigma_{x} k_{y}-\sigma_{y} k_{x}\right)=\alpha_{1} i\left(k_{-} \sigma_{+}-k_{+} \sigma_{-}\right),
$$

where $k_{ \pm} \equiv k_{x} \pm i k_{y}$ and $\sigma_{ \pm} \equiv\left(\sigma_{x} \pm i \sigma_{y}\right) / 2$. For the most common case of a (001) surface BIA has two contributions, the linear Dresselhaus term

$$
H_{D 1}=\beta_{1}\left(\sigma_{y} k_{y}-\sigma_{x} k_{x}\right)=-\beta_{1}\left(k_{+} \sigma_{+}+k_{-} \sigma_{-}\right),
$$

and the cubic Dresselhaus term

$$
\begin{aligned}
H_{D 3} & =\beta_{3}\left(\sigma_{x} k_{x} k_{y}^{2}-\sigma_{y} k_{y} k_{x}^{2}\right) \\
& =\beta_{3}\left[k_{-}\left(k_{+}^{2}-k_{-}^{2}\right) \sigma_{+}+k_{+}\left(k_{-}^{2}-k_{+}^{2}\right) \sigma_{-}\right] .
\end{aligned}
$$

In a quantum well with well width $w$ we have approximately $\beta_{1}=\beta_{3}(\pi / w)^{2}$ (Ref. 67). This implies that the linear Dresselhaus term often dominates in more narrow electron systems with smaller density (i.e., small Fermi wave vector), whereas the cubic Dresselhaus term may 
dominate in wider quantum wells with a larger density. Experiments can be designed to focus on these different regimes. Even in the latter case we typically remain in the electric quantum limit, where only the lowest subband of the quantized motion in $z$ direction is occupied. ${ }^{36}$ In the following we will focus on this regime.

For 2D heavy-hole systems SO coupling due to SIA is dominated by the cubic Rashba Hamiltonian,

$$
\begin{aligned}
H_{R 3} & =\alpha_{3}\left[k_{y}\left(k_{y}^{2}-3 k_{x}^{2}\right) \sigma_{x}+k_{x}\left(k_{x}^{2}-k_{y}^{2}\right) \sigma_{y}\right] \\
& =\alpha_{1} i\left(k_{+}^{3} \sigma_{-}-k_{-}^{3} \sigma_{+}\right) .
\end{aligned}
$$

BIA in 2D heavy-hole systems on a (001) surface contains the $k$-linear term

$$
H_{D 1^{\prime}}=\gamma_{1}\left(\sigma_{x} k_{x}+\sigma_{y} k_{y}\right)=\gamma_{1}\left(k_{+} \sigma_{-}+k_{-} \sigma_{+}\right),
$$

and the cubic Dresselhaus term

$$
\begin{aligned}
H_{D 3^{\prime}} & =\gamma_{3}\left(k_{x}^{2}+k_{y}^{2}\right)\left(\sigma_{x} k_{x}+\sigma_{y} k_{y}\right) \\
& =\gamma_{3}\left(k_{+}^{2} k_{-} \sigma_{-}+k_{-}^{2} k_{+} \sigma_{+}\right) .
\end{aligned}
$$

For the terms cubic in $k$, we restricted ourselves to the dominant contributions due to SIA and BIA. $H_{D 1^{\prime}}$ and $H_{D 3^{\prime}}$ are often comparable in magnitude.

\section{EFFECTIVE POSITION OPERATOR}

The SO interaction appears when transforming from the Dirac to the Pauli equation by means of the FoldyWouthuysen transformation. ${ }^{54}$ Under this transformation, the position operator in spin- $1 / 2$ systems becomes

$$
\hat{\boldsymbol{r}}_{\text {phys }}=\hat{\boldsymbol{r}}+\hat{\boldsymbol{r}}_{\text {so }}
$$

where the SO part $\hat{\boldsymbol{r}}_{\text {so }}$ is expressed in terms of the vector $\boldsymbol{\sigma}$ of Pauli spin matrices. We refer to $\hat{\boldsymbol{r}}_{\text {so }}$ as the anomalous position operator.

The general form for the anomalous position operator, valid for both $2 \mathrm{D}$ electron and $2 \mathrm{D}$ hole systems, is

$$
\hat{\boldsymbol{r}}_{s o}=\lambda \boldsymbol{\sigma} \times \boldsymbol{\omega}_{\boldsymbol{k}}
$$

where $\lambda$ and $\boldsymbol{\omega}_{\boldsymbol{k}}$ are different for electrons and holes. For $2 \mathrm{D}$ electrons $\boldsymbol{\omega}_{\boldsymbol{k}}=\boldsymbol{k}$, and

$$
\hat{\boldsymbol{r}}_{s o}=\lambda_{1} \boldsymbol{\sigma} \times \boldsymbol{k}
$$

assuming $\lambda_{1} k_{F}^{2} \ll 1$. For $2 \mathrm{D}$ hole systems the correction to the position operator has the form

$$
\hat{\boldsymbol{r}}_{s o}=\lambda_{3} \boldsymbol{\sigma} \times \boldsymbol{\omega}_{\boldsymbol{k} 3},
$$

where $\boldsymbol{\omega}_{\boldsymbol{k} 3}=k^{3}(\cos 3 \theta, \sin 3 \theta, 0)$, assuming $\lambda_{3} k_{F}^{6} \ll 1$.

Consider a general scalar potential $V(\hat{\boldsymbol{r}})$. Under the Foldy-Wouthuysen transformation it transforms to $V\left(\hat{\boldsymbol{r}}_{\text {phys }}\right)$, which, to first order in $\hat{\boldsymbol{r}}_{s o}$, takes the form

$$
V\left(\hat{\boldsymbol{r}}_{p h y s}\right)=V(\hat{\boldsymbol{r}})+\frac{1}{2}\left[\nabla V(\hat{\boldsymbol{r}}) \cdot \hat{\boldsymbol{r}}_{s o}+\hat{\boldsymbol{r}}_{s o} \cdot \nabla V(\hat{\boldsymbol{r}})\right] .
$$

Therefore, as a result of this transformation, both the potential due to an applied electric field and the impurity scattering potential acquire spin-dependent terms.

Let $U(\boldsymbol{r})$ denote the scattering potential, which represents elastic scattering off charged impurities and static defects (but not phonons or electrons)

$$
U(\boldsymbol{r})=\sum_{I} \bar{U}\left(\boldsymbol{r}-\boldsymbol{R}_{I}\right)
$$

where $\boldsymbol{R}_{I}$ indexes the random locations of the impurities and the scattering potential due to a single impurity is denoted by $\bar{U}(\boldsymbol{r})$. In Fourier space, the matrix elements of $U(\boldsymbol{r})$ are

$$
U_{\boldsymbol{k} \boldsymbol{k}^{\prime}}=\bar{U}_{\boldsymbol{k} \boldsymbol{k}^{\prime}} \sum_{I} e^{i\left(\boldsymbol{k}-\boldsymbol{k}^{\prime}\right) \cdot \boldsymbol{R}_{I}}
$$

and the potential due to a single impurity is written as

$$
\bar{U}_{\boldsymbol{k} \boldsymbol{k}^{\prime}}=\mathcal{U}_{\boldsymbol{k} \boldsymbol{k}^{\prime}} \mathbb{1}+\mathcal{V}_{\boldsymbol{k} \boldsymbol{k}^{\prime}}
$$

where $\mathcal{U}_{\boldsymbol{k} \boldsymbol{k}^{\prime}}$ represents the matrix element of the potential due to a single impurity between plane waves, while $\mathcal{V}_{\boldsymbol{k} \boldsymbol{k}^{\prime}}$ is the spin-dependent part arising from $\hat{\boldsymbol{r}}_{\text {so }}$. Both have units of energy $\times$ volume. The strength of the disorder potential is characterized by the impurity density $n_{i}$. The matrix elements of the spin-dependent part of the impurity potential in reciprocal space are

$$
\mathcal{V}_{\boldsymbol{k} \boldsymbol{k}^{\prime}}=-\frac{i \lambda}{2} \boldsymbol{\sigma} \cdot\left(\boldsymbol{\omega}_{\boldsymbol{k}} \times \boldsymbol{k}^{\prime}-\boldsymbol{\omega}_{\boldsymbol{k}^{\prime}} \times \boldsymbol{k}\right) \mathcal{U}_{\boldsymbol{k} \boldsymbol{k}^{\prime}}
$$

In 2D the spin dependent term in $\mathcal{V}_{\boldsymbol{k} \boldsymbol{k}^{\prime}}$ points out of the plane for both electron and hole systems.

Interaction with a static, uniform external electric field $\boldsymbol{E}$ is contained in

$$
\begin{aligned}
H_{E \boldsymbol{k} \boldsymbol{k}^{\prime}} & =(e \boldsymbol{E} \cdot \hat{\boldsymbol{r}})_{\boldsymbol{k} \boldsymbol{k}^{\prime}} \mathbb{1}+e\left(\boldsymbol{E} \cdot \hat{\boldsymbol{r}}_{s o}\right)_{\boldsymbol{k} \boldsymbol{k}} \delta_{\boldsymbol{k} \boldsymbol{k}^{\prime}} \\
& =i e \boldsymbol{E} \cdot \frac{\partial}{\partial \boldsymbol{k}} \delta\left(\boldsymbol{k}-\boldsymbol{k}^{\prime}\right) \mathbb{1}+\frac{1}{2} \boldsymbol{\sigma} \cdot \boldsymbol{\Delta}_{\boldsymbol{k}} \delta_{\boldsymbol{k} \boldsymbol{k}^{\prime}} .
\end{aligned}
$$

with $\mathbb{1}$ the identity matrix in spin space, and $\boldsymbol{\Delta}_{\boldsymbol{k}}$ arises from the anomalous position operator. ${ }^{68}$ From Eq. (12),

$$
\boldsymbol{\Delta}_{\boldsymbol{k}}=2 e \lambda \boldsymbol{\omega}_{\boldsymbol{k}} \times \boldsymbol{E} .
$$

It follows from the preceding discussion that $\boldsymbol{\Delta}_{\boldsymbol{k}}$ has different forms in electron and hole systems.

The anomalous position operator accounts for impurity SO coupling and for band structure SO coupling due to SIA. To see the latter, consider the SO coupling due to the full potential $V_{\text {tot }}$ acting on the system. In a $2 \mathrm{D}$ system we can divide $V_{t o t}=V_{\text {ext }}+V_{Q W}+U$, where $V_{\text {ext }}$ is the applied electric field, $V_{Q W}$ the $z$-direction confinement, and $U$ the impurity potential introduced above. The total potential $V_{t o t}$ gives rise to SO coupling, which in reciprocal space is contained in

$$
H_{s o, \boldsymbol{k}}=\lambda_{n} \boldsymbol{\sigma} \cdot \boldsymbol{k} \times \boldsymbol{\nabla}\left(V_{e x t}+U\right)+\lambda_{n} \boldsymbol{\sigma} \cdot \boldsymbol{k} \times \hat{\boldsymbol{z}}\left(\frac{\partial V_{Q W}}{\partial z}\right) .
$$


In the second term we can incorporate the average $\left\langle\partial V_{Q W} / \partial z\right\rangle$ over the quantum well into an effective SO constant $\alpha$, giving the Rashba SO coupling. ${ }^{81}$ This clarifies the relationship between $\alpha$ and $\lambda$ and shows that, knowing the form of the Rashba Hamiltonian in a certain system, one can deduce the form of $\hat{\boldsymbol{r}}_{s o}$ in that system.

The full Hamiltonian is $H_{\boldsymbol{k}}^{\text {tot }}=H_{0 \boldsymbol{k}}+H_{E \boldsymbol{k} \boldsymbol{k}^{\prime}}+U_{\boldsymbol{k} \boldsymbol{k}^{\prime}}$. The spin current operator $\hat{j}_{j}^{i}$ corresponding to spin component $i$ flowing in the direction $j$ is

$$
\hat{j}_{j}^{i}=\frac{\hbar^{2} k_{j}}{2 m} \sigma_{i}
$$

In addition to the contribution from the band Hamiltonian, the velocity operator has two additional terms, discussed in detail in Ref. 62. The first stems from the spin-dependent interaction with the external electric field $H_{E \boldsymbol{k} \lambda}$, while the second arises from the spin-dependent term $\mathcal{V}_{\boldsymbol{k} \boldsymbol{k}^{\prime}}$ in the impurity potential. These two cancel, as they represent the net force acting on the system. ${ }^{62}$ They will not be explicitly considered in what follows.

\section{KINETIC EQUATION}

The formalism presented here parallels that originally formulated in Refs. 8,9. The Liouville equation for the density operator $\hat{\rho}$ is projected onto the basis $\{|\boldsymbol{k}\rangle\}$, with $\rho_{\boldsymbol{k} \boldsymbol{k}^{\prime}}=f_{\boldsymbol{k}} \delta_{\boldsymbol{k} \boldsymbol{k}^{\prime}}+g_{\boldsymbol{k} \boldsymbol{k}^{\prime}}$, where $g_{\boldsymbol{k} \boldsymbol{k}^{\prime}}$ is off-diagonal in $\boldsymbol{k}$, and all quantities are matrices in spin space. These satisfy

$$
\begin{aligned}
\frac{d f_{\boldsymbol{k}}}{d t}+\frac{i}{\hbar}\left[\hat{H}_{0}, \hat{f}\right]_{\boldsymbol{k} \boldsymbol{k}} & =-\frac{i}{\hbar}[\hat{U}, \hat{g}]_{\boldsymbol{k} \boldsymbol{k}} \\
\frac{d g_{\boldsymbol{k} \boldsymbol{k}^{\prime}}}{d t}+\frac{i}{\hbar}\left[\hat{H}_{0}, \hat{g}\right]_{\boldsymbol{k} \boldsymbol{k}^{\prime}} & =-\frac{i}{\hbar}[\hat{U}, \hat{f}]_{\boldsymbol{k} \boldsymbol{k}^{\prime}}-\frac{i}{\hbar}[\hat{U}, \hat{g}]_{\boldsymbol{k} \boldsymbol{k}^{\prime}}
\end{aligned}
$$

We focus on variations which are slow on the scale of the momentum relaxation time, and solve for $g_{\boldsymbol{k} \boldsymbol{k}^{\prime}}$ as an expansion in the impurity potential, which can be performed to any desired order. Very generally $f_{\boldsymbol{k}}$ satisfies

$$
\frac{d f_{\boldsymbol{k}}}{d t}+\frac{i}{\hbar}\left[\hat{H}_{0}, \hat{f}\right]_{\boldsymbol{k} \boldsymbol{k}}+\hat{J}\left(f_{\boldsymbol{k}}\right)=0
$$

The total scattering term $\hat{J}\left(f_{\boldsymbol{k}}\right)=\hat{J}_{\text {Born }}\left(f_{\boldsymbol{k}}\right)+\hat{J}_{s s}\left(f_{\boldsymbol{k}}\right)$, where in the first Born approximation

$$
\hat{J}_{\text {Born }}\left(f_{\boldsymbol{k}}\right)=\frac{1}{\hbar^{2}}\left\langle\int_{0}^{\infty} d t^{\prime}\left[\hat{U}, e^{-i \hat{H}_{0} t^{\prime} / \hbar}[\hat{U}, \hat{f}] e^{i \hat{H}_{0} t^{\prime} / \hbar}\right]\right\rangle_{\boldsymbol{k} \boldsymbol{k}}
$$

and $\langle\ldots\rangle$ represents averaging over impurity configurations. In the second Born approximation we obtain the additional skew scattering term

$$
\hat{J}_{s s}\left(f_{\boldsymbol{k}}\right)=-\frac{i}{\hbar^{3}}\left\langle\int_{0}^{\infty} d t^{\prime} \int_{0}^{\infty} d t^{\prime \prime}\left[\hat{U}, e^{-i \hat{H}_{0} t^{\prime} / \hbar}\left[\hat{U}, e^{-i \hat{H}_{0} t^{\prime \prime} / \hbar}[\hat{U}, \hat{f}] e^{i \hat{H}_{0} t^{\prime \prime} / \hbar}\right] e^{i \hat{H}_{0} t^{\prime} / \hbar}\right]\right\rangle_{\boldsymbol{k} \boldsymbol{k}} .
$$

We expand $\hat{J}_{\text {Born }}\left(f_{\boldsymbol{k}}\right)$ in $\Omega_{\boldsymbol{k}}$ and $\lambda$. We retain the leading term plus terms to first order in $\Omega_{\boldsymbol{k}}$, first order in $\lambda$, and the second-order term in $\Omega_{\boldsymbol{k}} \lambda$. Thus $\hat{J}_{\text {Born }}\left(f_{\boldsymbol{k}}\right)$ can be written as a perturbation expansion in $\Omega_{\boldsymbol{k}}$ and $\lambda$ in the form

$$
\hat{J}_{\mathrm{Born}}\left(f_{\boldsymbol{k}}\right)=\hat{J}_{0}\left(f_{\boldsymbol{k}}\right)+\hat{J}_{\Omega}\left(f_{\boldsymbol{k}}\right)+\hat{J}_{\mathrm{sj}}\left(f_{\boldsymbol{k}}\right)+\hat{J}_{\Omega \lambda}\left(f_{\boldsymbol{k}}\right) .
$$

The leading term in $\hat{J}_{\text {Born }}\left(f_{\boldsymbol{k}}\right)$ is the scalar $\hat{J}_{0}\left(f_{\boldsymbol{k}}\right)$, which is the customary Born-approximation scattering term appearing in the Boltzmann equation. It is found by taking Eq. (26) and considering only the scalar parts of $\hat{H}_{0}$ (i.e. $H_{\text {kin }}$ ) and $\hat{U}$ (i.e. $\left.\mathcal{U}_{\boldsymbol{k} \boldsymbol{k}^{\prime}}\right)$, and in $2 \mathrm{D}$ takes the form

$$
\hat{J}_{0}\left(f_{\boldsymbol{k}}\right)=\frac{n_{i} m^{*}}{\hbar^{3}} \int \frac{d \theta^{\prime}}{2 \pi}\left|\mathcal{U}_{\boldsymbol{k} \boldsymbol{k}^{\prime}}\right|^{2}\left(f_{\boldsymbol{k}}-f_{\boldsymbol{k}^{\prime}}\right)
$$

Next, we have the term in $\hat{J}_{\text {Born }}\left(f_{\boldsymbol{k}}\right)$ to first order in $\Omega_{\boldsymbol{k}}$ (i.e. due to band-structure SO coupling), which is found by considering the spin-dependent part of $\hat{H}_{0}$ and the scalar part of $\hat{U}$. It gives rise to a well-known scattering term, referred to here as $\hat{J}_{\Omega}\left(f_{\boldsymbol{k}}\right) \cdot{ }^{8,9}$ We only require its action on the scalar part of the density matrix, $n_{\boldsymbol{k}}$, given by

$$
\hat{J}_{\Omega}\left(n_{\boldsymbol{k}}\right)=\frac{\pi}{\hbar} \int \frac{d^{2} k^{\prime}}{(2 \pi)^{2}}\left|\mathcal{U}_{\boldsymbol{k} \boldsymbol{k}^{\prime}}\right|^{2}\left(n_{\boldsymbol{k}}-n_{\boldsymbol{k}^{\prime}}\right) \boldsymbol{\sigma} \cdot\left(\boldsymbol{\Omega}_{\boldsymbol{k}}-\boldsymbol{\Omega}_{\boldsymbol{k}^{\prime}}\right) \frac{\partial}{\partial \varepsilon_{0}} \delta\left(\varepsilon_{0 \boldsymbol{k}}-\varepsilon_{0 \boldsymbol{k}^{\prime}}\right)
$$

This term is relevant only in determining the band-structure SO contribution to the spin current, which has been studied previously, and is not pertinent to the discussion presented in this work and will not be given. Following on, in the side-jump scattering term $\hat{J}_{s j}\left(f_{\boldsymbol{k}}\right)$ we take the scalar part of $\hat{H}_{0}$ and the spin-dependent part of $\hat{U}$. The electric field $\boldsymbol{E}$ is also finite in this term: without it $\hat{J}_{s j}\left(f_{\boldsymbol{k}}\right)$ would vanish. ${ }^{82}$ Because $\boldsymbol{E}$ is nonzero, $\hat{J}_{s j}\left(f_{\boldsymbol{k}}\right)$ acts on the equilibrium density matrix $f_{0 \boldsymbol{k}}$. It has two parts, which have been determined in Ref. 62. We use the notation of 
Ref. 62. We write $\hat{J}_{\mathrm{sj}}\left(n_{\boldsymbol{k}}\right)=\hat{J}_{\mathrm{sj}}^{a}\left(n_{\boldsymbol{k}}\right)+\hat{J}_{\mathrm{sj}}^{b}\left(n_{\boldsymbol{k}}\right)$. The first part of the side-jump scattering term, referred to as $\hat{J}_{\mathrm{sj}}^{a}\left(n_{\boldsymbol{k}}\right)$, arises from the change in the band energy due to the spin-dependent energy of interaction with $\boldsymbol{E}$

$$
\hat{J}_{\mathrm{sj}}^{a}\left(n_{\boldsymbol{k}}\right)=\frac{2 \pi n_{i}}{\hbar} \int \frac{d^{2} k^{\prime}}{(2 \pi)^{2}}\left|\mathcal{U}_{\boldsymbol{k} \boldsymbol{k}^{\prime}}\right|^{2}\left(n_{\boldsymbol{k}}-n_{\boldsymbol{k}^{\prime}}\right) \frac{1}{2} \boldsymbol{\sigma} \cdot\left(\boldsymbol{\Delta}_{\boldsymbol{k}}-\boldsymbol{\Delta}_{\boldsymbol{k}^{\prime}}\right) \frac{\partial}{\partial \varepsilon_{0 \boldsymbol{k}}} \delta\left(\varepsilon_{0 \boldsymbol{k}}-\varepsilon_{0 \boldsymbol{k}^{\prime}}\right) .
$$

The second part, $\hat{J}_{\mathrm{sj}}^{b}\left(n_{\boldsymbol{k}}\right)$, reflects the spin-dependent change in the carrier position during collisions

$$
\hat{J}_{\mathrm{sj}}^{b}\left(n_{\boldsymbol{k}}\right)=\frac{i n_{i} \pi e \boldsymbol{E}}{\hbar} \cdot \int \frac{d^{2} k^{\prime}}{(2 \pi)^{2}} \mathcal{U}_{\boldsymbol{k} \boldsymbol{k}^{\prime}}\left(\frac{\partial \mathcal{V}_{\boldsymbol{k}^{\prime} \boldsymbol{k}}}{\partial \boldsymbol{k}^{\prime}}+\frac{\partial \mathcal{V}_{\boldsymbol{k}^{\prime} \boldsymbol{k}}}{\partial \boldsymbol{k}}\right)\left(n_{\boldsymbol{k}}-n_{\boldsymbol{k}^{\prime}}\right) \frac{\partial}{\partial \varepsilon_{0 \boldsymbol{k}^{\prime}}} \delta\left(\varepsilon_{0 \boldsymbol{k}}-\varepsilon_{0 \boldsymbol{k}^{\prime}}\right)+h . c .
$$

Both parts of the side jump scattering term are $\propto \sigma_{z}$.

The scattering term $\hat{J}_{\Omega \lambda}\left(n_{\boldsymbol{k}}\right)$ reads

$$
\hat{J}_{\Omega \lambda}\left(n_{\boldsymbol{k}}\right)=\frac{\pi n_{i}}{\hbar} \int \frac{d^{d} k^{\prime}}{(2 \pi)^{d}}\left[\boldsymbol{\sigma} \cdot \boldsymbol{\Omega}_{\boldsymbol{k}^{\prime}}, \mathcal{V}_{\boldsymbol{k} \boldsymbol{k}^{\prime}}\right] \mathcal{U}_{\boldsymbol{k} \boldsymbol{k}^{\prime}}\left(n_{\boldsymbol{k}}-n_{\boldsymbol{k}^{\prime}}\right) \frac{\partial}{\partial \varepsilon_{0 \boldsymbol{k}}} \delta\left(\varepsilon_{0 \boldsymbol{k}}-\varepsilon_{0 \boldsymbol{k}^{\prime}}\right)
$$

The physical meaning of this term is as follows. During a scattering process, an incoming spin has a well-defined spin direction, given by $\boldsymbol{\Omega}_{\boldsymbol{k}}$, which represents the bandstructure SO coupling at wave vector $\boldsymbol{k}$. Because the scattering potential is also spin dependent, the incoming spin is rotated during scattering by an amount that is proportional to $\mathcal{V}_{\boldsymbol{k} \boldsymbol{k}^{\prime}}$, the impurity SO coupling. This scattering term therefore represents spin rotations during collisions induced by the impurity SO coupling, the rotation being evident from its commutator structure.

Even though we are doing perturbation theory to first order in the SO interaction terms $\lambda$ and $\Omega_{\boldsymbol{k}}$, spin preces- sion makes it necessary to include driving terms to order $\lambda \Omega_{\boldsymbol{k}}$, since these terms also yield contributions to the spin current $\propto \lambda$ only, i.e. to first order in the impurity SO coupling. The necessity of including terms $\propto \lambda \Omega_{k}$ will become apparent when we discuss explicitly the solution for $S_{E \boldsymbol{k}}$ introduced below, during which it will emerge that spin precession introduces a factor of $1 / \Omega_{k}$.

Beyond the first Born approximation we retain the leading term $\hat{J}_{s s}\left(f_{\boldsymbol{k}}\right)$, in which $\lambda$ is finite but the electric field $\boldsymbol{E}=0$, which is customarily responsible for skew scattering. ${ }^{68}$ To first order in $\lambda$, the real part of this term reduces to

$$
\hat{J}_{s s}\left(n_{\boldsymbol{k}}\right)=-\frac{3 \pi^{2} n_{i} \lambda}{\hbar} \int \frac{d^{2} k^{\prime}}{(2 \pi)^{2}} \int \frac{d^{2} k^{\prime \prime}}{(2 \pi)^{2}} \mathcal{U}_{\boldsymbol{k} \boldsymbol{k}^{\prime}} \mathcal{U}_{\boldsymbol{k}^{\prime} \boldsymbol{k}^{\prime \prime}} \mathcal{U}_{\boldsymbol{k}^{\prime \prime} \boldsymbol{k}} \boldsymbol{\sigma} \cdot\left(\boldsymbol{\omega}_{\boldsymbol{k}} \times \boldsymbol{k}^{\prime}-\boldsymbol{\omega}_{\boldsymbol{k}^{\prime}} \times \boldsymbol{k}\right)\left(n_{\boldsymbol{k}^{\prime}}-n_{\boldsymbol{k}^{\prime \prime}}\right) \delta\left(\varepsilon_{0 \boldsymbol{k}}-\varepsilon_{0 \boldsymbol{k}^{\prime \prime}}\right) \delta\left(\varepsilon_{0 \boldsymbol{k}}-\varepsilon_{0 \boldsymbol{k}^{\prime}}\right)
$$

In $2 \mathrm{D}$ systems, in which both $\boldsymbol{k}$ and $\boldsymbol{\omega}_{\boldsymbol{k}}$ are in the $x y$-plane, the skew scattering term is $\propto \sigma_{z}$.

\section{NON-EQUILIBRIUM DENSITY MATRIX}

In a constant uniform electric field $\boldsymbol{E}$ the density matrix is $f_{\boldsymbol{k}}=f_{0 \boldsymbol{k}}+f_{E \boldsymbol{k}}$. The equilibrium density matrix is given by

$$
f_{0 \boldsymbol{k}}=\frac{1}{2}\left[f_{\mathrm{FD}}\left(\varepsilon_{\boldsymbol{k}+}\right)+f_{\mathrm{FD}}\left(\varepsilon_{\boldsymbol{k}-}\right)\right]+\frac{1}{2}\left[f_{\mathrm{FD}}\left(\varepsilon_{\boldsymbol{k}+}\right)-f_{\mathrm{FD}}\left(\varepsilon_{\boldsymbol{k}-}\right)\right] \boldsymbol{\sigma} \cdot \hat{\boldsymbol{\Omega}}_{\boldsymbol{k}},
$$

with $f_{\mathrm{FD}}$ the Fermi-Dirac distribution function, while $f_{E \boldsymbol{k}}$ is due to $\boldsymbol{E}$. To first order in $\boldsymbol{E}$ the correction $f_{E \boldsymbol{k}}$ satisfies

$$
\frac{\partial f_{E \boldsymbol{k}}}{\partial t}+\frac{i}{\hbar}\left[H_{\boldsymbol{k}}, f_{E \boldsymbol{k}}\right]+\hat{J}\left(f_{E \boldsymbol{k}}\right)=\frac{e \boldsymbol{E}}{\hbar} \cdot \frac{\partial f_{0 \boldsymbol{k}}}{\partial \boldsymbol{k}}-\frac{i}{2 \hbar}\left[\boldsymbol{\sigma} \cdot \boldsymbol{\Delta}_{\boldsymbol{k}}, f_{0 \boldsymbol{k}}\right] .
$$

The term $(e \boldsymbol{E} / \hbar) \cdot\left(\partial f_{0 \boldsymbol{k}} / \partial \boldsymbol{k}\right)$ corresponds to the usual streaming term in the Boltzmann equation. The second term on the RHS of Eq. (36) appears due to the anomalous position operator and is $\propto \lambda$.
We write $f_{k}=n_{k} \mathbb{1}+S_{k}$, where $S_{k}$ is a $2 \times 2$ Hermitian matrix, and correspondingly $f_{E \boldsymbol{k}}=n_{E \boldsymbol{k}} \mathbb{1}+S_{E \boldsymbol{k}}$ and $f_{0 \boldsymbol{k}}=n_{0 \boldsymbol{k}} \mathbb{1}+S_{0 \boldsymbol{k}}$. The expectation values of the spin current operator is found from $S_{E \boldsymbol{k}}$. The term 
$(e \boldsymbol{E} / \hbar) \cdot\left(\partial f_{0 \boldsymbol{k}} / \partial \boldsymbol{k}\right)$ may be decomposed into a scalar part $(e \boldsymbol{E} / \hbar) \cdot\left(\partial n_{0 \boldsymbol{k}} / \partial \boldsymbol{k}\right)$ and a spin-dependent part $(e \boldsymbol{E} / \hbar) \cdot$ $\left(\partial S_{0 \boldsymbol{k}} / \partial \boldsymbol{k}\right)$. The spin-dependent part has been studied previously, ${ }^{8,9}$ and is responsible for current-induced spin polarizations and spin currents arising from the bandstructure SO coupling. It will not be discussed in this work.

The non-equilibrium correction to the scalar part of the density matrix, $n_{E \boldsymbol{k}}$, is determined from

$$
\frac{\partial n_{E \boldsymbol{k}}}{\partial t}+\hat{J}_{0}\left(n_{E \boldsymbol{k}}\right)=\frac{e \boldsymbol{E}}{\hbar} \cdot \frac{\partial n_{0 \boldsymbol{k}}}{\partial \boldsymbol{k}} .
$$

The solution to this equation is well known, and reads $n_{E \boldsymbol{k}}=\left(e \boldsymbol{E} \tau_{p} / \hbar\right) \cdot\left(\partial n_{0 \boldsymbol{k}} / \partial \boldsymbol{k}\right)$, with $\tau_{p}$ the momentum relaxation time. Once this solution is found, the spindependent scattering terms $\hat{J}_{s s}, \hat{J}_{s j}$ and $\hat{J}_{\Omega \lambda}$ act on $n_{E \boldsymbol{k}}$ and produce additional effective driving terms for $S_{E \boldsymbol{k}}$. (The method used is the same as in Ref. 69.)

We seek the solution for $S_{E \boldsymbol{k}}$ to first order in $\lambda$ which we denote by $S_{E \boldsymbol{k} \lambda}$. Specifically, including the contribution due to $\boldsymbol{\Delta}_{\boldsymbol{k}}$ from Eq. (36), it is found from

$$
\frac{\partial S_{E \boldsymbol{k} \lambda}}{\partial t}+\frac{i}{\hbar}\left[H_{\boldsymbol{k}}, S_{E \boldsymbol{k} \lambda}\right]+\hat{J}_{0}\left(S_{E \boldsymbol{k} \lambda}\right)=-\hat{J}_{s s}\left(n_{E \boldsymbol{k}}\right)-\hat{J}_{s j}\left(n_{E \boldsymbol{k}}\right)-\hat{J}_{\Omega \lambda}\left(n_{E \boldsymbol{k}}\right)-\frac{i}{\hbar}\left[H_{E \boldsymbol{k} \lambda}, S_{0 \boldsymbol{k}}\right]
$$

We specialize to short-range impurities henceforth, without loss of generality. The potential of a single impurity in Fourier space is written as

$$
\begin{aligned}
& \bar{U}_{\boldsymbol{k} \boldsymbol{k}^{\prime}}=\mathcal{U} \mathbb{1}+\mathcal{V}_{\boldsymbol{k} \boldsymbol{k}^{\prime}} \\
& \mathcal{V}_{\boldsymbol{k} \boldsymbol{k}^{\prime}}=-\frac{i \lambda \mathcal{U}}{2} \boldsymbol{\sigma} \cdot\left(\boldsymbol{\omega}_{\boldsymbol{k}} \times \boldsymbol{k}^{\prime}-\boldsymbol{\omega}_{\boldsymbol{k}^{\prime}} \times \boldsymbol{k}\right),
\end{aligned}
$$

where the Fourier transform $\mathcal{U}_{\boldsymbol{k} \boldsymbol{k}^{\prime}}$ has become the constant $\mathcal{U}$. We write $\hat{J}_{0}\left(f_{\boldsymbol{k}}\right)=\left(f_{\boldsymbol{k}}-\overline{f_{\boldsymbol{k}}}\right) / \tau$, with the overline denoting an angular average over the directions of $\hat{\boldsymbol{k}}$, which in $2 \mathrm{D}$ indicates an average over the polar angle $\theta$,

$$
\bar{X} \equiv \int \frac{d \theta}{2 \pi} X
$$

and the momentum relaxation time $\tau_{p} \equiv \tau$, given by

$$
\frac{1}{\tau}=\frac{n_{i} m^{*} \mathcal{U}^{2}}{\hbar^{3}}
$$

We discuss the driving terms in more detail. Firstly,

$$
-\hat{J}_{s s}\left(n_{E \boldsymbol{k}}\right)=\frac{3 n_{i} \lambda m^{* 2}|\mathcal{U}|^{3}}{4 \hbar^{5}} \boldsymbol{\sigma} \cdot \overline{\left(\boldsymbol{\omega}_{\boldsymbol{k}} \times \boldsymbol{k}^{\prime}-\boldsymbol{\omega}_{\boldsymbol{k}^{\prime}} \times \boldsymbol{k}\right) n_{E \boldsymbol{k}^{\prime}}},
$$

where the overline denotes averaging over $\theta^{\prime}$ and the integration over $k^{\prime}$ forces $k^{\prime}=k$. We have established that this term is $\propto \sigma_{z}$, and inspection of Eq. (42) reveals that this term is an odd function of $\boldsymbol{k}$.

The anomalous interaction with $\boldsymbol{E}$ gives rise to two driving terms. The first arises from the side-jump scattering term, which was determined in Ref. 62. For both electrons and holes this takes the form

$$
-\hat{J}_{s j}\left(n_{E \boldsymbol{k}}\right)=-\frac{1}{\tau} \boldsymbol{\sigma} \cdot \boldsymbol{\Delta}_{\boldsymbol{k}} \delta\left(\varepsilon_{0 \boldsymbol{k}}-\varepsilon_{F}\right) .
$$

This term is also odd in $\boldsymbol{k} \cdot{ }^{83}$ An additional driving term comes from the commutator of $\frac{1}{2} \boldsymbol{\sigma} \cdot \boldsymbol{\Delta}_{\boldsymbol{k}}$ with the density matrix. Given that $\boldsymbol{\Delta}_{\boldsymbol{k}}$ is already first-order in $\boldsymbol{E}$ we require only the equilibrium density matrix $f_{0 \boldsymbol{k}}$. We expand $f_{0 \boldsymbol{k}}=f_{\mathrm{FD}}\left(\varepsilon_{\boldsymbol{k}}\right) \mathbb{1}+(\hbar / 2) \boldsymbol{\sigma} \cdot \boldsymbol{\Omega}_{\boldsymbol{k}} \frac{\partial f_{\mathrm{FD}}\left(\varepsilon_{\boldsymbol{k}}\right)}{\partial \varepsilon_{\boldsymbol{k}}}$, where the first term is a scalar, and at temperature $T=0$ we can write

$$
-\frac{i}{\hbar}\left[H_{E \boldsymbol{k}}^{s j}, f_{0 \boldsymbol{k}}\right]=\frac{1}{2} \delta\left(\varepsilon_{0 \boldsymbol{k}}-\varepsilon_{F}\right) \boldsymbol{\sigma} \cdot \boldsymbol{\Omega}_{\boldsymbol{k}} \times \boldsymbol{\Delta}_{\boldsymbol{k}}
$$

Notice that this term is zero in the absence of spin precession, when $f_{0 \boldsymbol{k}}$ is a scalar and the commutator vanishes.

The remaining driving term is $-\hat{J}_{\Omega \lambda}\left(n_{E \boldsymbol{k}}\right)$. For $2 \mathrm{D}$ electron systems,

$$
-\hat{J}_{\Omega \lambda}\left(n_{E \boldsymbol{k}}\right)=\frac{i \pi \lambda n_{i}|\mathcal{U}|^{2}}{\hbar} \int \frac{d^{2} k^{\prime}}{(2 \pi)^{2}}\left[\boldsymbol{\sigma} \cdot \boldsymbol{\Omega}_{\boldsymbol{k}^{\prime}}, \boldsymbol{\sigma} \cdot \boldsymbol{k} \times \boldsymbol{k}^{\prime}\right]\left(n_{E \boldsymbol{k}}-n_{E \boldsymbol{k}^{\prime}}\right) \frac{\partial}{\partial \varepsilon_{0 \boldsymbol{k}}} \delta\left(\varepsilon_{0 \boldsymbol{k}}-\varepsilon_{0 \boldsymbol{k}^{\prime}}\right) .
$$

For 2D hole systems,

$$
-\hat{J}_{\Omega \lambda}\left(n_{E \boldsymbol{k}}\right)=\frac{i \lambda \pi n_{i}|\mathcal{U}|^{2}}{2 \hbar} \int \frac{d^{2} k^{\prime}}{(2 \pi)^{2}}\left[\boldsymbol{\sigma} \cdot \boldsymbol{\Omega}_{\boldsymbol{k}^{\prime}}, \boldsymbol{\sigma} \cdot\left(\boldsymbol{\omega}_{\boldsymbol{k} 3} \times \boldsymbol{k}^{\prime}-\boldsymbol{\omega}_{\boldsymbol{k}^{\prime} 3} \times \boldsymbol{k}\right)\right]\left(n_{E \boldsymbol{k}}-n_{E \boldsymbol{k}^{\prime}}\right) \frac{\partial}{\partial \varepsilon_{0 \boldsymbol{k}}} \delta\left(\varepsilon_{0 \boldsymbol{k}}-\varepsilon_{0 \boldsymbol{k}^{\prime}}\right) .
$$

\section{SOLUTION OF THE KINETIC EQUATION}

We summarize first the general solution to the kinetic equation for short range impurities and weak momentum scattering. We denote the driving terms generically by 
$\mathcal{D}_{E \boldsymbol{k} \lambda}$ in this section. Let the component $i$ of the spin operator be denoted by $\hat{s}_{i}=(\hbar / 2) \sigma_{i}$. The spin density is $\operatorname{Tr} \rho \hat{s}_{i}=\operatorname{Tr} \bar{\rho} \hat{s}_{i}$, where the overbar denotes an angular average as above, thus $\bar{\rho}$ is the isotropic part of the density matrix. Similarly, the spin current operator $\hat{j}_{j}^{i}$ has been defined in Eq. (23). Because it is odd in $\boldsymbol{k}$ its expectation value yields $\operatorname{Tr} \rho \hat{j}_{j}^{i}=\operatorname{Tr}(\rho-\bar{\rho}) \hat{j}_{j}^{i}$. Consequently, the isotropic part of the spin density matrix determines the spin density, while the anisotropic part of the density matrix determines the spin current. It is therefore convenient to divide the spin density matrix into $S_{E \boldsymbol{k} \lambda}=\overline{S_{E \boldsymbol{k} \lambda}}+T_{E \boldsymbol{k} \lambda}$, the isotropic part being $\overline{S_{E \boldsymbol{k} \lambda}}$ (which gives the spin density) and the anisotropic part $T_{E \boldsymbol{k} \lambda}$ (which gives the spin current). From the quantum Liouville equation, we obtain a set of coupled equations for $\overline{S_{E \boldsymbol{k} \lambda}}$ and $T_{E \boldsymbol{k} \lambda}$ for short-range impurities, which are solved rigorously in Appendix A. Here we just quote the solution. Letting $\mathcal{D}_{E \boldsymbol{k} \lambda}=\frac{1}{2} \boldsymbol{\sigma} \cdot \boldsymbol{d}_{E \boldsymbol{k} \lambda}$, we find for $\Omega_{\boldsymbol{k}} \tau \gg 1$

$$
T_{E \boldsymbol{k} \lambda}=\frac{1}{2} \boldsymbol{\sigma} \cdot\left(\frac{\hat{\boldsymbol{\Omega}}_{\boldsymbol{k}}}{\Omega_{\boldsymbol{k}}}\right) \times\left[\boldsymbol{d}_{E \boldsymbol{k} \lambda}+\overline{\mathcal{A}}^{-1}\left(\overline{\boldsymbol{d}_{E \boldsymbol{k} \lambda}}-\overline{\mathcal{A} \boldsymbol{d}_{E \boldsymbol{k} \lambda}}\right)\right],
$$

where the (dimensionless) matrix $\mathcal{A}$ is given by $\mathcal{A}_{i j}=$ $\left(\delta_{i j}-\hat{\Omega}_{i} \hat{\Omega}_{j}\right)$, and $T_{E \boldsymbol{k} \lambda}$ as found in Eq. (47) gives the spin current in the weak momentum scattering limit. Finally, we take the electric field $\boldsymbol{E} \| \hat{\boldsymbol{x}}$, the spin-Hall conductivity is defined by $j_{y}^{z}=\sigma_{y x}^{z} E_{x}$, and we abbreviate the spin-Hall conductivity due to $T_{E \boldsymbol{k} \lambda}$ simply by $\sigma_{\lambda}$.

The appearance of the $\Omega_{k}$ in the denominator of Eq. (47) is a crucial feature of this solution. It demonstrates the need to retain scattering terms $\propto \lambda \Omega_{k}$ that are formally of second order in the SO coupling.

\section{A. Skew scattering and side-jump scattering}

We recall that, as shown in Eqs. (42) and (43), both $\hat{J}_{\mathrm{ss}}\left(n_{E \boldsymbol{k}}\right)$ and $\hat{J}_{\mathrm{sj}}\left(n_{E \boldsymbol{k}}\right)$ are odd in $\boldsymbol{k}$. Therefore the driving terms due to $\hat{J}_{\mathrm{ss}}\left(n_{E \boldsymbol{k} \boldsymbol{k}}\right)$ and $\hat{J}_{\mathrm{sj}}\left(n_{E \boldsymbol{k}}\right)$ yield corrections to $S_{E \boldsymbol{k} \lambda}$ that are even in $\boldsymbol{k}$. Since the spin current operator $\hat{j}_{j}^{i}$ is odd in $\boldsymbol{k}$, simple power counting in Eq. (47) reveals that $\hat{J}_{\mathrm{ss}}\left(n_{E \boldsymbol{k}}\right)$ and $\hat{J}_{\mathrm{sj}}\left(n_{E \boldsymbol{k}}\right)$ do not give a spin current in the weak momentum scattering regime. We can develop a physical understanding of this fact. In the absence of spin precession, skew scattering and side-jump scattering separate up-spins from down-spins. When band structure SO interactions are present, each spin precesses about an effective magnetic field which depends on $\boldsymbol{k}$, thus it is not conserved. Electrons are driven by the external field and collide with impurities, with up-spins scattering predominantly in one direction and down-spins predominantly in the other direction. The spins then travel towards the edges of the sample, yet they are subjected to the action of the band structure SO effective field, which causes them to precess. Upon arriving at the edge the spins are completely randomized. Therefore, very generally, side-jump scattering and
Table I: $\hat{\boldsymbol{r}}_{s o}$ contributions to the SHE in units of $n_{e} e \lambda$ for $\Omega \tau \gg 1$. Here $e^{-}\left(h^{+}\right)$stands for electrons (holes), while "band SO" abbreviates "band-structure SO".

\begin{tabular}{ccccc}
\hline \hline system & band SO & $\boldsymbol{\Delta}_{\boldsymbol{k}}$ & $\sigma_{\lambda}^{\text {prec }}$ & $\sigma_{\lambda}^{\text {sct }}$ \\
\hline$e^{-}$ & $R 1$ & $\boldsymbol{k}$ & $1 / 2$ & $-1 / 2$ \\
$e^{-}$ & $D 1$ & $\boldsymbol{k}$ & $1 / 2$ & $-1 / 2$ \\
$e^{-}$ & $D 3$ & $\boldsymbol{k}$ & $1 / 2$ & $-1 / 4$ \\
$h^{+}$ & $R 3$ & $\boldsymbol{k}^{3}$ & 0 & 0 \\
$h^{+}$ & $D 1^{\prime}$ & $\boldsymbol{k}^{3}$ & 0 & 0 \\
$h^{+}$ & $D 3^{\prime}$ & $\boldsymbol{k}^{3}$ & 0 & 0 \\
\hline \hline
\end{tabular}

skew scattering do not give rise to a spin current in $2 \mathrm{D}$ systems.

\section{B. Anomalous spin precession from electric field}

Using Eq. 47, we have a term in the density matrix

$$
S_{E \boldsymbol{k} \lambda}^{\mathrm{prec}}=-\frac{1}{2} \boldsymbol{\sigma} \cdot \boldsymbol{\Delta}_{\boldsymbol{k}} \frac{\Omega_{\boldsymbol{k}}^{2} \tau^{2}}{1+\Omega_{\boldsymbol{k}}^{2} \tau^{2}} \delta\left(\varepsilon_{0 \boldsymbol{k}}-\varepsilon_{F}\right)
$$

In the weak momentum scattering limit $\Omega_{\boldsymbol{k}} \tau \gg 1$ this result is independent of the form of the band structure $\mathrm{SO}$ interaction, and can be easily obtained from the driving term in Eq. (44). We have given (in this subsection alone) a result valid beyond the weak momentum scattering limit so as to emphasize this apparent independence is only an artifact of this limit. For electron systems in this limit, the spin-Hall conductivity due to this term is,

$$
\sigma_{\lambda}^{\text {prec }}=\frac{n_{e} e \lambda}{2}
$$

where $n_{e}$ is the electron density. In the weak momentum scattering limit this term is also independent of $\tau$. In $2 \mathrm{D}$ electron systems it recovers the nonzero contribution to the SHE originally found by Tse and Das Sarma ${ }^{63}$ and subsequently by Raimondi and Schwab. ${ }^{66}$ In $2 \mathrm{D}$ hole systems it is easy to check that $\sigma_{\lambda}^{\text {prec }}=0$.

The origin of this contribution to the SHE will be elucidated in Sec. VII, but one remark is in order here. The spin-Hall conductivity $\sigma_{\lambda}^{\text {prec }}$ found in Eq. (49) has the opposite sign to that found in Refs. $62,63,66$ for the same orientation of the electric field. One should therefore not think of $\sigma_{\lambda}^{\text {prec }}$ as a surviving side-jump term, but a qualitatively new term due to $\hat{\boldsymbol{r}}_{s o}$ altogether, which we identify with a spin precession mechanism with no counterpart in systems without band structure SO coupling.

\section{Anomalous spin precession from impurities}

The last piece in the puzzle is the driving term $\hat{J}_{\Omega \lambda}\left(n_{E \boldsymbol{k}}\right)$, which needs to be studied independently for each model. We denote the contribution of this term to $\sigma_{\lambda}$ by $\sigma_{\lambda}^{\text {sct }}$. Once found, this term is added to $\sigma_{\lambda}^{\text {prec }}$ to give $\sigma_{\lambda}$, which yields the total SHE due to $\hat{\boldsymbol{r}}_{s o}$. 


\section{Linear Rashba and Dresselhaus $S O$}

For linear Rashba band structure SO coupling $H_{R 1}$

$$
\hat{J}_{\Omega \lambda}\left(n_{E \boldsymbol{k}}\right)=\frac{2 e \alpha \lambda m k}{\hbar^{3}} \boldsymbol{E} \cdot \hat{\boldsymbol{k}} \boldsymbol{\sigma} \cdot \hat{\boldsymbol{\theta}} \delta\left(k-k_{F}\right) .
$$

The spin-Hall conductivity due to this driving term is

$$
\sigma_{\lambda}^{\mathrm{sct}}=-\frac{n_{e} e \lambda}{2} .
$$

This term exactly cancels $\sigma_{\lambda}^{\text {prec }}$. The same holds for the linear Dresselhaus SO interaction $H_{D 1}$.

\section{Cubic Dresselhaus SO}

In general $\sigma_{\lambda}^{\text {prec }}$ and $\sigma_{\lambda}^{\text {sct }}$ do not cancel. We consider next a 2DEG in which the band structure SO coupling is described by the cubic Dresselhaus Hamiltonian $H_{D 3}$. In this case, the scattering term $\hat{J}_{\Omega \lambda}^{D 3}\left(n_{E \boldsymbol{k}}\right)$ is given by

$$
\begin{aligned}
\hat{J}_{\Omega \lambda}\left(n_{E \boldsymbol{k}}\right)= & -\frac{m e \beta \lambda k^{3}}{\hbar^{3}} \boldsymbol{E} \cdot \hat{\boldsymbol{k}}(\boldsymbol{\sigma} \cdot \hat{\boldsymbol{\theta}} \sin 2 \theta-\boldsymbol{\sigma} \cdot \hat{\boldsymbol{k}} \cos 2 \theta) \\
& \times \delta\left(k-k_{F}\right) .
\end{aligned}
$$

This gives a significant contribution to the spin-Hall current,

$$
\sigma_{\lambda}^{\text {sct }}=-\frac{n_{e} e \lambda}{4} .
$$

The remaining term due to $\hat{\boldsymbol{r}}_{s o}$ is $\sigma_{\lambda}^{\text {prec }}$, and thus in the weak momentum scattering limit

$$
\sigma_{\lambda}=\frac{n_{e} e \lambda}{4} .
$$

The magnitude of the SHE conductivity due to the band structure SO coupling (the band-structure SHE) in the 2D cubic Dresselhaus model has been calculated to be $-e / 16 \pi$ in the clean limit. ${ }^{70}$ Therefore the total SHE conductivity, including that due to band structure $\mathrm{SO}$, is

$$
\sigma_{y x}^{z} \approx-\frac{e}{16 \pi}+\frac{n_{e} e \lambda}{4} .
$$

The term due to band-structure $\mathrm{SO}$ is densityindependent, whereas the anomalous spin precession term in the SHE is linear in $n_{e}$. These are the only two terms in the clean limit when the band structure SO coupling is described by the cubic Dresselhaus model.

The cubic Dresselhaus term $H_{D 3}$ is strong in a wide quantum well at high electron density $n_{e}$. However, the full Hamiltonian for such a system in general involves both linear and cubic Dresselhaus SO terms, $H_{D 1}$ and $H_{D 3}$, whose interplay is nontrivial. We discuss the full conditions required for experimental observation of anomalous spin precession in this complex case in Sec. VIII.

\section{Hole systems}

It is easily seen that in 2D hole systems both $\sigma_{\lambda}^{\text {prec }}$ and $\sigma_{\lambda}^{\text {sct }}$ are zero. For holes, $\boldsymbol{\Delta}_{\boldsymbol{k}}$ can be found from Eqs. (14) and (20). Substituting this into Eq. 48, we find that the spin-Hall current averages to zero over directions in momentum space. In $\hat{J}_{\Omega \lambda}\left(n_{E \boldsymbol{k}}\right)$, in all cases studied, terms $\propto e^{ \pm 3 i \theta}$ cause the angular integral to vanish. Therefore, in $2 \mathrm{D}$ hole systems

$$
\sigma_{\lambda}^{\mathrm{sct}}=0
$$

There is thus no contribution to the SHE due to anomalous spin precession in 2D hole systems.

\section{DISCUSSION}

To summarize, $\sigma_{\lambda}=0$ in $2 \mathrm{D}$ hole systems, while in $2 \mathrm{D}$ electron systems in the weak momentum scattering regime it can be written as

$$
\sigma_{\lambda}=\frac{n_{e} e \lambda}{2}+\sigma_{\lambda}^{s c t}
$$

The results for the total SHE due to $\hat{\boldsymbol{r}}_{s o}$ are summarized in Table I. Interestingly, $\sigma_{\lambda}$ can be nonzero, even though that is only true in one out of the several situations studied explicitly in this work.

We have argued previously that $\sigma_{\lambda}^{\text {prec }}$ should be thought of not as a surviving side-jump term, but a qualitatively new term, which is not present in systems without band structure SO. We demonstrate that this term is related to spin precession induced by both band structure SO and $\hat{\boldsymbol{r}}_{s o}$. The electric field $\boldsymbol{E}$ gives rise to an additional SO effective field $\boldsymbol{\Delta}_{\boldsymbol{k}} \| \hat{\boldsymbol{z}}$-direction. The band structure SO effective field $\boldsymbol{\Omega}_{\boldsymbol{k}}$ is in the plane. We examine spin precession in the total effective magnetic field $\boldsymbol{\Omega}_{\boldsymbol{k}}$ and $\boldsymbol{\Delta}_{\boldsymbol{k}}$, redefining $\boldsymbol{\Omega}_{\boldsymbol{k}} \rightarrow \tilde{\boldsymbol{\Omega}}_{\boldsymbol{k}}$, with

$$
\tilde{\Omega}_{k}=\Omega_{k}+\Delta_{k} \text {. }
$$

Let $\tilde{\boldsymbol{\Omega}}_{\boldsymbol{k}}=\left(\tilde{\Omega}_{x}, 0,0\right)$ and turn on $\boldsymbol{E}$ adiabatically, generating a small $\tilde{\Omega}_{z} \ll \tilde{\Omega}_{x}$. We study the Heisenberg equation of motion for the spin (Bloch) vector $s$, which reads $d \boldsymbol{s} / d t=\tilde{\boldsymbol{\Omega}} \times \boldsymbol{s}$, in a clean system. The spin is taken initially to be parallel to $\tilde{\Omega}_{x}$. In component form

$$
\begin{aligned}
\frac{d s_{x}}{d t} & =-\tilde{\Omega}_{z} s_{y} \\
\frac{d s_{y}}{d t} & =\tilde{\Omega}_{z} s_{x}-\tilde{\Omega}_{x} s_{z} \\
\frac{d s_{z}}{d t} & =\tilde{\Omega}_{x} s_{y} .
\end{aligned}
$$

One can take the time derivative one more time and solve the equations exactly, yet the physics is evident from Eq. (59b). Since $\boldsymbol{s}(t=0)=\left(s_{x}, 0,0\right)$ and $s_{y}$ is initially zero, 
$s_{y}$ should remain zero at all times. Setting $d s_{y} / d t$ in the steady state we obtain

$$
s_{z}=s_{x}\left(\frac{\tilde{\Omega}_{z}}{\tilde{\Omega}_{x}}\right) .
$$

The explanation is as follows: $s_{y}$ is initially 0 and must remain 0. When $\boldsymbol{E}$ is turned on an additional component $\tilde{\Omega}_{z}$ is generated, which makes $s_{x}$ precess and gives a small contribution to $s_{y}$. To cancel this, $s_{z}$ must develop a small out-of-plane component, which precesses around $\tilde{\Omega}_{x}$, and gives the exact opposite contribution to $s_{y}$. The extra $s_{z}$ density has opposite signs for the two halves of the Fermi surface, giving rise to a net spin-Hall current. The argument presented here shows that $\hat{\boldsymbol{r}}_{\text {so }}$ gives rise to a spin-Hall current even in a clean system. We refer to this process as anomalous spin precession.

This argument can be generalized to explain anomalous spin precession in a disordered system as well. This can be done by replacing $\boldsymbol{E} \rightarrow \boldsymbol{E}+\boldsymbol{\nabla} U(\boldsymbol{r})$, and understanding this to represent the total local electric field. We thus reproduce both anomalous spin precession terms - the one due to the external electric field and the one due to the impurity potential. Both terms give an effective magnetic field out of the plane of the quantum well, modifying the intrinsic SO spin precession.

Equation (47) is valid for weak momentum scattering. Appendix A shows that in the strong momentum scattering regime $\overline{S_{E \boldsymbol{k} \lambda}}$ diverges. Physically, this is because we are using $\boldsymbol{\Omega}_{\boldsymbol{k}}$ as our reference, and projections parallel and perpendicular to it become ill-defined as $\boldsymbol{\Omega}_{\boldsymbol{k}} \rightarrow 0$. In this limit Dyakonov-Perel spin relaxation is no longer active, and there is no spin relaxation at all. We demonstrate in Appendix A that the divergence in the strong momentum scattering regime is cured by the introduction of the Elliott-Yafet spin relaxation time $\tau_{\mathrm{EY}}$, which is also related to $\boldsymbol{V}_{\boldsymbol{k n}^{\prime}}$. Nevertheless, in order to be consistent one would have to formulate the entire theory up to order $\lambda^{2}$, which is beyond the scope of this paper.

In deriving $\sigma_{\lambda}$ we have assumed for simplicity that the scattering potential is short ranged. We do not expect the results to change qualitatively for long-range impurities. Firstly, we have shown that $\sigma_{\lambda}^{\text {prec }}$ is independent of scattering in weak momentum scattering limit and is traced to a mechanism unrelated to disorder. Secondly, although for a general potential the anisotropic terms in $\mathcal{U}_{\boldsymbol{k} \boldsymbol{k}^{\prime}}$ will depend on the form of the potential, as will $\sigma_{\lambda}^{\text {sct }}$, we do not expect cancellation between $\sigma_{\lambda}^{\text {sct }}$ and $\sigma_{\lambda}^{\text {prec', }}$, even though $\sigma_{\lambda}^{\text {sct }}$ may have a different numerical value from that determined. Finally, past experience with the SHE shows that important cancellations, such as that of the SHE due to Rashba band structure SO coupling, tend to have a fundamental origin ${ }^{71}$ and are independent of whether the scattering potential is short-range or long-range. ${ }^{8,9}$

\section{EXPERIMENTAL OBSERVATION}

We have argued that the anomalous spin precession contribution to the SHE in general is finite. For example it survives in $2 \mathrm{D}$ electron gases in which the $\mathrm{SO}$ interaction is described by the cubic Dresselhaus term $\left(H_{D 3}\right)$. In Sec. VIC 2 we calculated the anomalous spin precession contribution to the SHE conductivity using purely the cubic Dresselhaus model. We now discuss the experimental conditions required for the observation of anomalous spin precession in a realistic sample.

For the anomalous spin precession contribution to the SHE to be observable it must ideally overwhelm the band structure contribution. Here we focus on two common semiconductor materials with strong SO coupling in the conduction band, InAs and InSb, and estimate the magnitude of the anomalous spin precession as well as the band structure contributions to the SHE in these materials. The constant $\lambda$ for InAs and InSb can be found in Table 6.6 in Ref. 67 (in the notation used in this paper, $\left.\lambda=r_{41}^{6 c 6 c} / e\right)$.

The situation is complicated by the fact that in realistic 2D samples both the linear and the cubic Dresselhaus terms, $H_{D 1}$ and $H_{D 3}$, are present. Having noted in Sec. II that $\beta_{1} \simeq \beta_{3}(\pi / w)^{2}$, the total SO Hamiltonian is

$$
H=\frac{\beta_{3} \pi^{2}}{w^{2}}\left(\sigma_{y} k_{y}-\sigma_{x} k_{x}\right)+\beta_{3}\left(\sigma_{x} k_{x} k_{y}^{2}-\sigma_{y} k_{y} k_{x}^{2}\right)
$$

The ratio $\pi /\left(k_{F} w\right)$ determines the relative magnitudes of $H_{D 1}$ and $H_{D 3}$. However, in order to have only one subband occupied it is necessary that $\pi /\left(k_{F} w\right) \geq 1$.

We showed in Sec. VIB that $\sigma_{\lambda}^{\text {prec }}$ is the same in the clean limit independently of the form of the band structure spin-orbit coupling. On the other hand, the contributions of the linear and cubic Dresselhaus terms, $H_{D 1}$ and $H_{D 3}$, to $\sigma_{\lambda}^{\text {sct }}$ are not simply additive, and their interplay is nontrivial. Therefore, the calculation of $\sigma_{\lambda}^{s c t}$ presented in Sec. VIC 2 needs to be repeated for the complicated case of $H=H_{D 1}+H_{D 3}$. This is done here analytically, except that the final results require a series of lengthy numerical integrations which can be performed using a symbolic algebra package. The results for $\sigma_{\lambda}^{\text {prec }}$ and $\sigma_{\lambda}^{\text {sct }}$ are summarized in Table II, as well as Fig. 1 .

The band structure contribution to the SHE for the case $H=H_{D 1}+H_{D 3}$ has been evaluated in Ref. 70. In Fig. 1 of that reference it was shown that the band structure SHE is a non-monotonic function of the parameter $\pi /\left(k_{F} w\right)$, where $\pi / w$ in our paper corresponds to the parameter $a$ in Ref. 70. In fact, the band structure SHE conductivity varies strongly as a function of this parameter and it changes sign at a critical value. It is however independent of $\beta_{3}$ in the clean limit, as is customary in $2 \mathrm{D}$ electron gases.

We consider a high-mobility quantum well with a number density $n_{e}=5 \times 10^{12} \mathrm{~cm}^{-2}$ for concreteness, a density commonly encountered in transport experiments. We focus on values of $w$ for which $\pi /\left(k_{F} w\right)$ is comprised between 1.0 (the widest well) and 1.4. 
Table II: Anomalous spin precession contributions to the spinHall conductivity in a $2 \mathrm{D}$ electron gas in a cubic crystal, with band-structure spin-orbit described by $H=H_{D 1}+H_{D 3}$, all in units of $n_{e} e \lambda$. In the last column $\sigma_{\lambda}=\sigma_{\lambda}^{\text {prec }}+\sigma_{\lambda}^{\text {sct }}$.

\begin{tabular}{cccc}
\hline \hline$\pi /\left(k_{F} w\right)$ & $\sigma_{\lambda}^{\text {prec }}$ & $\sigma_{\lambda}^{\text {sct }}$ & $\sigma_{\lambda}$ \\
& & & \\
\hline 1.00 & 0.5 & -0.315 & 0.185 \\
1.05 & 0.5 & -0.338 & 0.162 \\
1.10 & 0.5 & -0.357 & 0.143 \\
1.15 & 0.5 & -0.374 & 0.126 \\
1.20 & 0.5 & -0.386 & 0.114 \\
1.25 & 0.5 & -0.398 & 0.102 \\
1.30 & 0.5 & -0.407 & 0.093 \\
1.35 & 0.5 & -0.416 & 0.084 \\
1.40 & 0.5 & -0.423 & 0.077 \\
\hline \hline
\end{tabular}

The band structure contribution including both linear and cubic terms is read off from Fig. 1 of Ref. 70 and is the same for InAs and InSb. Our Eq. (55) (the pure cubic case) corresponds to $a=0$ in Eq. (16) of Ref. 70. Note also that, in the notation of Ref. 70, $e$ denotes the electron charge, whereas in our notation the electron charge is $-e$ : hence the seemingly opposite sign of the first term of our Eq. (55) compared to the corresponding formula of Ref. 70. When $\pi /\left(k_{F} w\right)=1.0$, the band structure contribution is $\approx 0.8 \times e /(16 \pi) \approx 0.016 e$ and, referring to Table II, we find the anomalous spin precession contribution to be $\approx 0.185 n_{e} e \lambda$. When $\pi /\left(k_{F} w\right)=1.4$, the band structure contribution decreases to $\approx 0.2 \times e /(16 \pi) \approx$ $0.004 e$, and the anomalous spin precession contribution to $\approx 0.077 n_{e} e \lambda$.

We consider first InAs, for which $\lambda=117 \AA^{2}$. At $\pi /\left(k_{F} w\right)=1.0$, with the value of $n_{e}$ specified above, we find the anomalous spin precession term to be $\approx 0.01 e$, which is just over half the size of the band structure term. At $\pi /\left(k_{F} w\right)=1.4$, the anomalous spin precession term is $0.0045 e$, marginally larger than the band structure term. In InAs therefore the band structure term is dominant in this parameter range.

In InSb, on the other hand, $\lambda=523 \AA^{2}$. At $\pi /\left(k_{F} w\right)=$ 1.0 , with the value of $n_{e}$ given above, we find the anomalous spin precession term to be $\approx 0.05 e$, three times larger than the band structure term. At $\pi /\left(k_{F} w\right)=1.4$, the anomalous spin precession term is $0.02 e$, five times larger than the band structure term. Thus, in InSb the anomalous spin precession is dominant in this parameter range.

We conclude that the most promising system for the observation of anomalous spin precession is the $2 \mathrm{D}$ electron gas in InSb. In the range $1.0 \leq \pi /\left(k_{F} w\right) \leq 1.4$ the anomalous spin precession provides the dominant contribution to the spin-Hall effect. At the lower end of this range, the overall SHE signal is stronger, and anomalous spin precession accounts for approximately three quarters of the SHE conductivity. At the upper end, although the

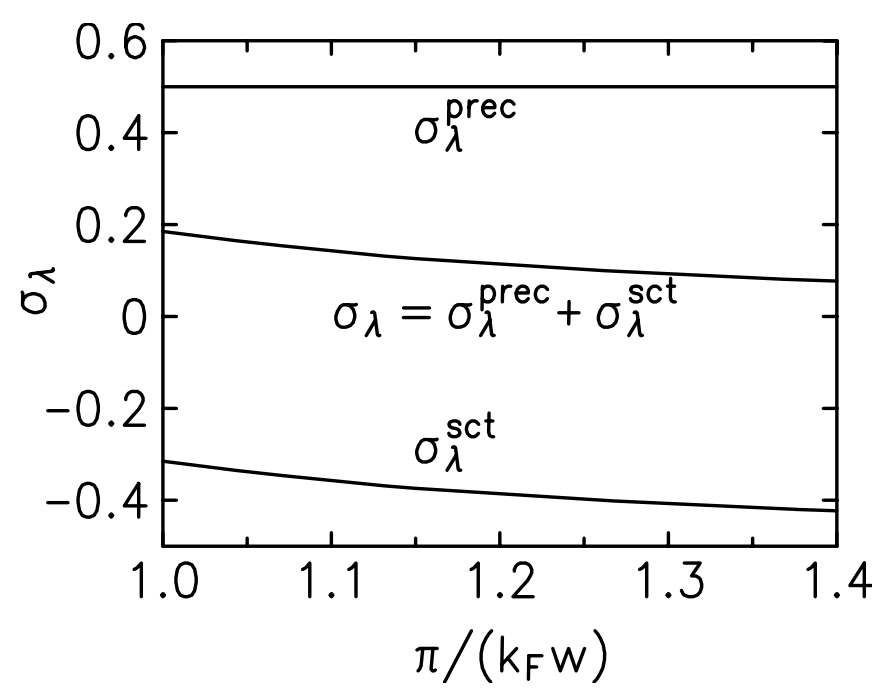

Figure 1: Anomalous spin precession contributions in a 2D electron gas with band structure spin-orbit coupling described by $H=H_{D 1}+H_{D 3}$ as a function of the parameter $\pi /\left(k_{F} w\right)$. On the vertical axis $\sigma_{\lambda}$ is measured in units of $n_{e} e \lambda$.

overall signal is weaker, anomalous spin precession accounts for approximately $5 / 6$ of the SHE conductivity.

\section{SUMMARY AND CONCLUSIONS}

We have determined all the contributions to the SHE due to the anomalous position operator $\hat{\boldsymbol{r}}_{s o}$ in $2 \mathrm{D}$ electron and hole systems. The SHE due to skew scattering and side-jump scattering vanishes in the presence of spin precession caused by the band structure SO coupling. Two additional contributions to the SHE exist due to $\hat{\boldsymbol{r}}_{\text {so }}$, one of which is scattering-dependent and one of which is due to anomalous spin precession under the action of $\hat{\boldsymbol{r}}_{s o}$ and the electric field. These two contributions cancel out in systems with band structure SO linear in $\boldsymbol{k}$, and are independently zero in 2D hole systems. However, the contribution due to anomalous spin precession survives in $2 \mathrm{D}$ electron systems with a significant cubic Dresselhaus term, i.e., for wide quantum wells with high electron densities, and is dominant under certain circumstances in InSb. Anomalous spin precession can therefore be detected in such a system.

A full account of the SHE in 2D systems must include the lengthy calculation of the electric field contribution to the skew scattering term, plus the band structure SO correction to that term. Moreover, in this work we have only considered heterostructures grown along the main crystal axes. Finally, the full answer will be known when the definition of the conserved spin current is taken into account, as has been done for the band-structure SHE. ${ }^{72}$ We reserve these studies for a future publication. 


\section{Acknowledgments}

We acknowledge insightful discussions with S. Das Sarma, O. P. Sushkov, Peter Schwab, Roberto Raimondi, Cosimo Gorini and W. K. Tse. DC was in part supported by the Chinese Academy of Sciences. E. M. H. was fi- nancially supported by the German Science Foundation DFG grant HA 5893/1-2 within the SPP 128. GV acknowledges support from NSF Grant No. DMR-1104788. Work at Argonne was supported by DOE BES under Contract No. DE-AC02-06CH11357.

\section{Appendix A: Decomposition of the spin density matrix into $\overline{S_{E k \lambda}}$ and $T_{E k \lambda}$}

¿From the quantum Liouville equation, we obtain for $\overline{S_{E \boldsymbol{k} \lambda}}$ and $T_{E \boldsymbol{k} \lambda}$ for short-range impurities

$$
\begin{aligned}
\frac{\partial \overline{S_{E \boldsymbol{k} \lambda}}}{\partial t}+\frac{i}{\hbar} \overline{\left[H, T_{E \boldsymbol{k} \lambda}\right]} & =\overline{\mathcal{D}_{E \boldsymbol{k} \lambda}} \\
\frac{\partial T_{E \boldsymbol{k} \lambda}}{\partial t}+\frac{i}{\hbar}\left[H, T_{E \boldsymbol{k} \lambda}\right]+\frac{T_{E \boldsymbol{k} \lambda}}{\tau} & =\left(\mathcal{D}_{E \boldsymbol{k} \lambda}-\overline{\mathcal{D}_{E \boldsymbol{k} \lambda}}\right)-\frac{i}{\hbar}\left[H, \overline{S_{E \boldsymbol{k} \lambda}}\right]+\frac{i}{\hbar} \overline{\left[H, T_{E \boldsymbol{k} \lambda}\right]} .
\end{aligned}
$$

On the RHS of Eq. (A1b) we substitute for $\frac{i}{\hbar} \overline{\left[H, T_{E \boldsymbol{k} \lambda}\right]}$ from Eq. (A1a). We rewrite Eqs. (A1) as

$$
\begin{aligned}
\frac{\partial \overline{S_{E \boldsymbol{k} \lambda}}}{\partial t}+\frac{i}{\hbar} \overline{\left[H_{\boldsymbol{k}}, T_{E \boldsymbol{k} \lambda}\right]} & =\overline{\mathcal{D}_{E \boldsymbol{k} \lambda}} \\
\frac{\partial T_{E \boldsymbol{k} \lambda}}{\partial t}+\frac{i}{\hbar}\left[H_{\boldsymbol{k}}, T_{E \boldsymbol{k} \lambda}\right]+\frac{T_{E \boldsymbol{k} \lambda}}{\tau} & =\mathcal{D}_{E \boldsymbol{k} \lambda}-\left(\frac{\partial \overline{S_{E \boldsymbol{k} \lambda}}}{\partial t}+\frac{i}{\hbar}\left[H_{\boldsymbol{k}}, \overline{S_{E \boldsymbol{k} \lambda}}\right]\right) .
\end{aligned}
$$

Defining $T_{E \boldsymbol{k} \lambda}=e^{-i H_{\boldsymbol{k}} t / \hbar} \tilde{T}_{E \boldsymbol{k} \lambda} e^{i H_{\boldsymbol{k}} t / \hbar}$ and $\overline{S_{E \boldsymbol{k} \lambda}}=e^{-i H_{\boldsymbol{k}} t / \hbar} \overline{S_{E \boldsymbol{k} \lambda}} e^{i H_{\boldsymbol{k}} t / \hbar}$, we can easily solve Eq. (A2b)

$$
\begin{aligned}
\frac{\partial \tilde{T}_{E \boldsymbol{k} \lambda}}{\partial t}+\frac{\tilde{T}_{E \boldsymbol{k} \lambda}}{\tau} & =e^{i H_{\boldsymbol{k}} t / \hbar} \mathcal{D}_{E \boldsymbol{k} \lambda} e^{-i H_{\boldsymbol{k}} t / \hbar}-\frac{\partial \overline{\bar{S}_{E \boldsymbol{k} \lambda}}}{\partial t} \\
\tilde{T}_{E \boldsymbol{k} \boldsymbol{\lambda}} & =-\tilde{\tilde{S_{E \boldsymbol{k} \lambda}}}+\int_{-\infty}^{t} d t^{\prime} e^{-\frac{\left(t-t^{\prime}\right)}{\tau}}\left[e^{i H t^{\prime} / \hbar} \mathcal{D}_{E \boldsymbol{k} \lambda} e^{-i H t^{\prime} / \hbar}+\frac{\tilde{S_{E \boldsymbol{k} \lambda}}}{\tau}\right] .
\end{aligned}
$$

where the last line was obtained by integration by parts. We can write $T_{E \boldsymbol{k} \lambda}$ (without the tilde) as

$$
T_{E \boldsymbol{k} \lambda}=-\overline{S_{E \boldsymbol{k} \lambda}}+\int_{0}^{\infty} d t^{\prime} e^{-\frac{t^{\prime}}{\tau}} e^{-i H t^{\prime} / \hbar}\left(\mathcal{D}_{E \boldsymbol{k} \lambda}+\frac{\overline{S_{E \boldsymbol{k} \lambda}}}{\tau}\right) e^{i H t^{\prime} / \hbar}
$$

Using $\overline{S_{E \boldsymbol{k} \lambda}}=\frac{1}{2} \boldsymbol{\sigma} \cdot \overline{\boldsymbol{s}_{E \boldsymbol{k} \lambda}}, \mathcal{D}_{E \boldsymbol{k} \lambda}=\frac{1}{2} \boldsymbol{\sigma} \cdot \boldsymbol{d}_{E \boldsymbol{k} \lambda}$ and $T_{E \boldsymbol{k} \lambda}=\frac{1}{2} \boldsymbol{\sigma} \cdot \boldsymbol{t}_{E \boldsymbol{k} \lambda}$, and carrying out the time integral

$$
\boldsymbol{t}_{E \boldsymbol{k} \lambda}=\hat{\boldsymbol{\Omega}}_{\boldsymbol{k}} \times\left(\boldsymbol{d}_{E \boldsymbol{k} \lambda}+\frac{\overline{\boldsymbol{s}}_{E \boldsymbol{k} \lambda}}{\tau}\right) \frac{\Omega_{\boldsymbol{k}} \tau^{2}}{1+\Omega_{\boldsymbol{k}}^{2} \tau^{2}}+\frac{\left(\boldsymbol{d}_{E \boldsymbol{k} \lambda} \tau\right)}{1+\Omega_{\boldsymbol{k}}^{2} \tau^{2}}+\text { additional terms }
$$

The physical interpretation of the terms appearing in Eq. A5 is as follows. The first term [containing $\hat{\Omega}_{\boldsymbol{k}} \times(\ldots)$ ] gives the full spin current when there is spin precession $\left(\Omega_{\boldsymbol{k}} \neq 0\right)$. The second term (containing $\left.\boldsymbol{d}_{E \boldsymbol{k} \lambda} \tau\right)$ recovers the spin current due to impurity SO coupling when there is no spin precession $\left(\Omega_{\boldsymbol{k}}=0\right)$. It vanishes in the weak momentum scattering limit $\Omega_{\boldsymbol{k}} \tau \gg 1$. Finally, the additional terms ensure that $\boldsymbol{t}_{E \boldsymbol{k} \lambda}$ averages to zero over directions in momentum space, but these terms give no spin current.

Let $\mathcal{A}_{i j}=\left(\delta_{i j}-\hat{\Omega}_{\boldsymbol{k} i} \hat{\Omega}_{\boldsymbol{k} j}\right)$, abbreviate $\mathcal{A} \overline{\boldsymbol{s}}_{E \boldsymbol{k} \boldsymbol{\lambda}} \equiv \mathcal{A}_{i j} \bar{s}_{E \boldsymbol{k} \lambda, j}$, and substitue Eq. (A5) into Eq.(A2a). In the steady state

$$
\frac{1}{\tau}\left[\overline{\left(\frac{\Omega_{\boldsymbol{k}}^{2} \tau^{2}}{1+\Omega_{\boldsymbol{k}}^{2} \tau^{2}}\right) \mathcal{A}}\right] \overline{\boldsymbol{s}_{E \boldsymbol{k} \lambda}}=\overline{\boldsymbol{d}_{E \boldsymbol{k} \lambda}}-\overline{\left(\frac{\Omega_{\boldsymbol{k}}^{2} \tau^{2}}{1+\Omega_{\boldsymbol{k}}^{2} \tau^{2}}\right) \mathcal{A} \boldsymbol{d}_{E \boldsymbol{k} \lambda}}+\overline{\frac{\left(\boldsymbol{\Omega}_{\boldsymbol{k}} \times \boldsymbol{d}_{E \boldsymbol{k} \lambda}\right) \tau}{1+\Omega_{\boldsymbol{k}}^{2} \tau^{2}}}
$$

using $\frac{i}{\hbar}\left[H_{\boldsymbol{k}}, \overline{S_{E \boldsymbol{k} \lambda}}\right]=-\frac{1}{2} \boldsymbol{\sigma} \cdot \boldsymbol{\Omega}_{\boldsymbol{k}} \times \overline{\boldsymbol{s}}_{E \boldsymbol{k} \lambda}$. For $\Omega_{\boldsymbol{k}} \tau \gg 1$ we obtain simply

$$
\begin{aligned}
\left(\frac{\overline{\mathcal{A}}}{\tau}\right) \overline{\boldsymbol{s}_{E \boldsymbol{k} \lambda}} & =\overline{\boldsymbol{d}_{E \boldsymbol{k} \lambda}}-\overline{\mathcal{A} \boldsymbol{d}_{E \boldsymbol{k} \lambda}} \\
\boldsymbol{t}_{E \boldsymbol{k} \lambda} & =\left(\frac{\hat{\boldsymbol{\Omega}}_{\boldsymbol{k}}}{\Omega_{\boldsymbol{k}}}\right) \times\left(\boldsymbol{d}_{E \boldsymbol{k} \lambda}+\frac{\overline{\boldsymbol{s}}_{E \boldsymbol{k} \lambda}}{\tau}\right)+\text { additional terms }
\end{aligned}
$$




\section{Appendix B: Elliott-Yafet spin relaxation time}

This derivation is for a general $S_{\boldsymbol{k}}$. Consider the scattering term in the Born approximation Eq. (26) up to second order in $\lambda$, and focus on its action on $S_{\boldsymbol{k}}$. In this term we may ignore the part of the time evolution operator $\propto \Omega_{\boldsymbol{k}}$. This scattering term is referred to as $\hat{J}_{\mathrm{EY}}\left(S_{\boldsymbol{k}}\right)$, and takes the form

$$
\begin{aligned}
\hat{J}_{\mathrm{EY}}\left(S_{\boldsymbol{k}}\right) & =\frac{\pi n_{i}}{\hbar} \int \frac{d^{2} k^{\prime}}{(2 \pi)^{2}} \mathcal{V}_{\boldsymbol{k} \boldsymbol{k}^{\prime}}\left(\mathcal{V}_{\boldsymbol{k}^{\prime} \boldsymbol{k}} S_{\boldsymbol{k}}-S_{\boldsymbol{k}^{\prime}} \mathcal{V}_{\boldsymbol{k}^{\prime} \boldsymbol{k}}\right) \delta\left(\varepsilon_{\boldsymbol{k}}-\varepsilon_{\boldsymbol{k}^{\prime}}\right)+h . c . \\
& =\frac{n_{i} m}{2 \hbar^{3}} \int_{0}^{2 \pi} \frac{d \theta^{\prime}}{2 \pi}\left(\left|\mathcal{V}_{\boldsymbol{k} \boldsymbol{k}^{\prime}}\right|^{2} S_{\boldsymbol{k}}-\mathcal{V}_{\boldsymbol{k} \boldsymbol{k}^{\prime}} S_{\boldsymbol{k}^{\prime}} \mathcal{V}_{\boldsymbol{k}^{\prime} \boldsymbol{k}}\right)+\text { h.c. }
\end{aligned}
$$

Bearing in mind that $\left|\mathcal{V}_{\boldsymbol{k} \boldsymbol{k}^{\prime}}\right|^{2}$ is a scalar, and in 2D systems $\mathcal{V}_{\boldsymbol{k} \boldsymbol{k}^{\prime}} \propto \sigma_{\boldsymbol{z}}$, the term $\mathcal{V}_{\boldsymbol{k} \boldsymbol{k}^{\prime}} \boldsymbol{S}_{\boldsymbol{k}^{\prime}} \mathcal{V}_{\boldsymbol{k}^{\prime} \boldsymbol{k}}$ has two possible forms:

$$
\mathcal{V}_{\boldsymbol{k} \boldsymbol{k}^{\prime}} S_{\boldsymbol{k}^{\prime}} \mathcal{V}_{\boldsymbol{k}^{\prime} \boldsymbol{k}}= \begin{cases}\left|\mathcal{V}_{\boldsymbol{k} \boldsymbol{k}^{\prime}}\right|^{2} S_{\boldsymbol{k}^{\prime}}, & \text { for } S_{\boldsymbol{k}^{\prime}} \propto \sigma_{z} \\ -\left|\mathcal{V}_{\boldsymbol{k} \boldsymbol{k}^{\prime}}\right|^{2} S_{\boldsymbol{k}^{\prime}}, & \text { for } S_{\boldsymbol{k}^{\prime}} \propto \sigma_{x}, \sigma_{y}\end{cases}
$$

so that

$$
\hat{J}_{\mathrm{EY}}\left(S_{\boldsymbol{k}}\right)=\frac{n_{i} m}{\hbar^{3}} \int_{0}^{2 \pi} \frac{d \theta^{\prime}}{2 \pi}\left|\mathcal{V}_{\boldsymbol{k} \boldsymbol{k}^{\prime}}\right|^{2}\left(S_{\boldsymbol{k}}-m_{z} S_{\boldsymbol{k}^{\prime}}\right)
$$

where $m_{z}=-1$ before $\sigma_{x}, \sigma_{y}$ and $m_{z}=1$ before $\sigma_{z}$. If $S_{\boldsymbol{k}} \propto \sigma_{z}$ the spin is out of the plane and is conserved during scattering, thus $\hat{J}_{\mathrm{EY}}\left(S_{\boldsymbol{k}}\right)$ gives just a correction to the momentum relaxation time. The change of sign for $S_{\boldsymbol{k}} \propto \sigma_{x}, \sigma_{y}$ is crucial. For short-range impurities, with $\left|\mathcal{V}_{\boldsymbol{k} \boldsymbol{k}^{\prime}}\right|^{2}=\lambda^{2} k^{4}|\mathcal{U}|^{2} \sin ^{2} \gamma$,

$$
\hat{J}_{\mathrm{EY}}\left(S_{\boldsymbol{k}}\right)=\frac{\lambda^{2} k^{4}}{2 \tau} \int_{0}^{2 \pi} \frac{d \theta^{\prime}}{2 \pi}\left(S_{\boldsymbol{k}}-m_{z} S_{\boldsymbol{k}^{\prime}}\right)(1-\cos 2 \gamma) .
$$

If we now write $S_{\boldsymbol{k}}=\overline{S_{\boldsymbol{k}}}+T_{\boldsymbol{k}}$, and define $\left(1 / \tau_{\mathrm{EY}}\right)=\lambda^{2} k^{4} / \tau$, then $\hat{J}_{\mathrm{EY}}\left(S_{\boldsymbol{k}}\right)$ simplifies to

$$
\hat{J}_{\mathrm{EY}}\left(S_{\boldsymbol{k}}\right)=\frac{S_{\boldsymbol{k}}-m_{z} \overline{S_{\boldsymbol{k}}}}{2 \tau_{\mathrm{EY}}}+\frac{m_{z}}{2 \tau_{\mathrm{EY}}} \int_{0}^{2 \pi} \frac{d \theta^{\prime}}{2 \pi} T_{\boldsymbol{k}^{\prime}} \cos 2 \gamma
$$

\section{Appendix C: $\tau_{\mathrm{EY}}$ cures divergence in $\overline{S_{E k \lambda}}$}

Equations (A7a) are correct as long as $\Omega \tau \gg 1$, otherwise $\overline{\boldsymbol{s}_{E \boldsymbol{k} \lambda}}$ found from Eq. (A6) diverges at small $\Omega \tau$. The way out of this dilemma is provided by the Elliott-Yafet spin relaxation time. Consider adding $\hat{J}_{\mathrm{EY}}\left(S_{\boldsymbol{k}}\right)$ to Eqs. (A1)

$$
\begin{aligned}
\frac{\partial \overline{S_{E \boldsymbol{k} \lambda}}}{\partial t}+\frac{i}{\hbar} \overline{\left[H, T_{E \boldsymbol{k} \lambda}\right]}+\frac{S_{E \boldsymbol{k} \lambda}-m_{z} \overline{S_{E \boldsymbol{k} \lambda}}}{2 \tau_{\mathrm{EY}}} & =\overline{\mathcal{D}_{\boldsymbol{k}}} \\
\frac{\partial T_{E \boldsymbol{k} \lambda}}{\partial t}+\frac{i}{\hbar}\left[H, T_{E \boldsymbol{k} \lambda}\right]+\frac{T_{E \boldsymbol{k} \lambda}}{\tau_{t o t}}+\hat{J}_{\mathrm{EY}}\left(T_{E \boldsymbol{k} \lambda}\right) & =\left(\mathcal{D}_{\boldsymbol{k}}-\overline{\mathcal{D}_{\boldsymbol{k}}}\right)-\frac{i}{\hbar}\left[H, \overline{S_{E \boldsymbol{k} \lambda}}\right]+\frac{i}{\hbar} \overline{\left[H, T_{E \boldsymbol{k} \lambda}\right]},
\end{aligned}
$$

where $1 / \tau_{\text {tot }}=1 / \tau+1 / \tau_{\mathrm{EY}}$. Since $\lambda k_{F}^{2} \ll 1$, the Elliott-Yafet spin relaxation time $\tau_{\mathrm{EY}} \gg \tau$, and the term containing the angular integral over $\theta^{\prime}$ is a very small correction to Eq. $(\mathrm{C} 1 \mathrm{~b})$, which may be neglected. The only change to the above formalism is an extra term in the equation for $\overline{S_{E \boldsymbol{k} \lambda}}$, which is nonzero for $\overline{S_{E \boldsymbol{k} \lambda}}$ in plane. The spin generated by an electric field is in-plane, so we can focus on this component, for which $m_{z}=-1$, and Eq. (A6) becomes

$$
\overline{\left(\frac{\Omega_{\boldsymbol{k}}^{2} \tau_{t o t}^{2}}{1+\Omega_{\boldsymbol{k}}^{2} \tau_{\text {tot }}^{2}}\right) \mathcal{A}} \overline{\frac{\boldsymbol{s}_{E \boldsymbol{k} \lambda}}{\tau_{t o t}}}+\frac{\overline{\boldsymbol{s}_{E \boldsymbol{k} \lambda}}}{\tau_{\mathrm{EY}}}=\overline{\boldsymbol{d}_{E \boldsymbol{k} \lambda}}-\overline{\mathcal{A} \boldsymbol{d}_{E \boldsymbol{k} \lambda}\left(\frac{\Omega_{\boldsymbol{k}}^{2} \tau_{t o t}^{2}}{1+\Omega_{\boldsymbol{k}}^{2} \tau_{\text {tot }}^{2}}\right)} .
$$

This cures the unphysical divergence at small $\Omega_{\boldsymbol{k}} \tau$. To see this, consider the simplest case, that of isotropic $\Omega_{\boldsymbol{k}}$,

$$
\overline{\boldsymbol{s}_{E \boldsymbol{k} \lambda}}=\frac{2 \overline{\boldsymbol{d}_{E \boldsymbol{k} \lambda}} \tau_{\text {tot }}\left(1+\Omega_{\boldsymbol{k}}^{2} \tau_{\text {tot }}^{2}\right)-2 \overline{\mathcal{A} \boldsymbol{d}_{E \boldsymbol{k} \lambda}} \Omega_{\boldsymbol{k}}^{2} \tau_{\text {tot }}^{3}}{\left[\Omega_{\boldsymbol{k}}^{2} \tau_{\text {tot }}^{2}+\left(2 \tau_{\text {tot }} / \tau_{\mathrm{EY}}\right)\left(1+\Omega_{\boldsymbol{k}}^{2} \tau_{\text {tot }}^{2}\right)\right]} .
$$


Clearly $\overline{\boldsymbol{s}_{E \boldsymbol{k} \lambda}} \rightarrow 0$ as $\Omega_{\boldsymbol{k}} \rightarrow 0$. Physically, the Elliott-Yafet spin relaxation time is needed to cure this divergence because projections parallel and perpendicular to $\boldsymbol{\Omega}_{\boldsymbol{k}}$ are ill-defined as $\boldsymbol{\Omega}_{\boldsymbol{k}} \rightarrow 0$.

1 M. I. Dyakonov and V. I. Perel, Phys. Lett. A 35, 459 (1971).

2 J. E. Hirsch, Phys. Rev. Lett. 83, 1834 (1999).

3 S. Zhang, Phys. Rev. Lett. 85, 393 (2000).

4 S. Murakami, N. Nagaosa, and S.-C. Zhang, Science 301, 1348 (2003).

5 J. Sinova, D. Culcer, Q. Niu, N. A. Sinitsyn, T. Jungwirth, and A. H. MacDonald, Phys. Rev. Lett. 92, 126603 (2004).

${ }^{6}$ H.-A. Engel, E. I. Rashba, and B. I. Halperin, in Handbook of Magnetism and Advanced Magnetic Materials, edited by H. Kronmüller and S. Parkin (Wiley, Chichester, UK, 2007), vol. V, pp. 2858-2877.

7 R. Winkler, in Handbook of Magnetism and Advanced Magnetic Meterials, edited by H. Kronmüller and S. Parkin (Wiley, Chichester, UK, 2007), vol. V, pp. 2830-2843.

8 D. Culcer and R. Winkler, Phys. Rev. B 76, 245322 (2007).

9 D. Culcer and R. Winkler, Phys. Rev. Lett 99, 226601 (2007).

10 W.-K. Tse, J. Fabian, I. Žutić, and S. Das Sarma, Phys. Rev. B 72, 241303 (2005).

11 A. Mal'shukov, L. Wang, C. Chu, and K. Chao, Phys. Rev. Lett. 95, 146601 (2005).

12 V. M. Galitski, A. A. Burkov, and S. Das Sarma, Phys. Rev. B 74, 115331 (2006).

13 L. Liu, O. J. Lee, T. J. Gudmundsen, D. C. Ralph, and R. A. Buhrman, arXiv:1110.6846 (2011).

14 M. W. Wu, J. H. Jiang, and M. Q. Weng, Phys. Rep. 493, 61 (2010).

15 J. Fabian, A. Matos-Abiague, C. Ertler, P. Stano, and I. Žutić, Acta Physica Slovaca 57, 565 (2007).

16 D. D. Awschalom and M. E. Flatté, Nature Physics 3, 153 (2007).

17 I. Žutić, J. Fabian, and S. Das Sarma, Rev. Mod. Phys. 76, 323 (2004).

18 B. Gu, J.-Y. Gan, N. Bulut, T. Ziman, G.-Y. Guo, N. Nagaosa, and S. Maekawa, Phys. Rev. Lett. 105, 086401 (2010).

19 T. Tanaka and H. Kontani, New Journal of Physics 11, 013023 (2009).

${ }^{20}$ G. Y. Guo, S. Murakami, T.-W. Chen, and N. Nagaosa, Phys. Rev. Lett. 100, 096401 (2008).

${ }^{21}$ M. Guigou, P. Recher, J. Cayssol, and B. Trauzettel, Phys. Rev. B 84, 094534 (2011).

22 J. L. Cheng and M. W. Wu, Journal of Physics: Condensed Matter 20, 085209 (2008).

23 V. K. Dugaev, M. Inglot, E. Y. Sherman, and J. Barnaś, Phys. Rev. B 82, 121310 (2010).

24 M. Gradhand, D. V. Fedorov, P. Zahn, and I. Mertig, Phys. Rev. B 81, 245109 (2010).

25 M. Gradhand, D. V. Fedorov, P. Zahn, and I. Mertig, Phys. Rev. Lett. 104, 186403 (2010).

26 M. I. Dyakonov, Phys. Rev. Lett. 99, 126601 (2007).

27 J. Shibata and H. Kohno, Phys. Rev. Lett. 102, 086603 (2009).

28 A. A. Kovalev, Y. Tserkovnyak, K. Výborný, and
J. Sinova, Phys. Rev. B 79, 195129 (2009)

29 S. Y. Liu, N. J. M. Horing, and X. L. Lei, Phys. Rev. B 74, 165316 (2006).

30 M. Duckheim, D. Loss, M. Scheid, K. Richter, I. Adagideli, and P. Jacquod, Phys. Rev. B 81, 085303 (2010).

31 E. Rashba, Semiconductors 42, 905 (2008).

32 P. Schwab, R. Raimondi, and C. Gorini, EPL (Europhysics Letters) 90, 67004 (2010).

33 S. A. Yang, H. Pan, Y. Yao, and Q. Niu, Phys. Rev. B 83, $125122(2011)$

34 Y. K. Kato, R. C. Myers, A. C. Gossard, and D. D. Awschalom, Science 306, 1910 (2004).

35 V. Sih, R. C. Myers, Y. K. Kato, W. H. Lau, A. C. Gossard, and D. D. Awschalom, Nature Physics 1, 31 (2005).

36 J. Wunderlich, B. Kaestner, J. Sinova, and T. Jungwirth, Phys. Rev. Lett. 94, 047204 (2005).

37 N. P. Stern, S. Ghosh, G. Xiang, M. Zhu, N. Samarth, and D. D. Awschalom, Phys. Rev. Lett. 97, 126603 (2006).

38 N. P. Stern, D. W. Steuerman, S. Mack, A. C. Gossard, and D. D. Awschalom, Nature Physics 4, 843 (2008).

39 C. Brune, A. Roth, E. G. Novik, M. Konig, H. Buhmann, E. M. Hankiewicz, W. Hanke, J. Sinova, and L. W. Molenkamp, Nat Phys 6, 448 (2010).

40 J.-H. Gao, J. Yuan, W.-Q. Chen, Y. Zhou, and F.-C. Zhang, Phys. Rev. Lett. 106, 057205 (2011).

41 B. Dora and R. Moessner, Phys. Rev. B 83, 073403 (2011).

42 D. Culcer, Physica E 44, 860 (2012).

43 E. Saitoh, M. Ueda, H. Miyajima, and G. Tatara., Appl. Phys. Lett. 88, 182509 (2006).

44 S. Valenzuela and M. Tinkham, Nature 442, 176 (2006).

45 T. Kimura, Y. Otani, T. Sato, S. Takahashi, and S. Maekawa, Phys. Rev. Lett. 98, 156601 (2007).

46 T. Seki, Y. Hasegawa, S. Mitani, S. Takahashi, H. Imamura, S. Maekawa, J. Nitta, and K. Takanashi, Nat Mater 7, 125 (2008).

47 B. Gu, I. Sugai, T. Ziman, G. Y. Guo, N. Nagaosa, T. Seki, K. Takanashi, and S. Maekawa, Phys. Rev. Lett. 105, 216401 (2010).

48 O. Mosendz, V. Vlaminck, J. E. Pearson, F. Y. Fradin, G. E. W. Bauer, S. D. Bader, and A. Hoffmann, Phys. Rev. B 82, 214403 (2010).

49 L. K. Werake, B. A. Ruzicka, and H. Zhao, Phys. Rev. Lett. 106, 107205 (2011).

50 Y. Niimi, M. Morota, D. H. Wei, C. Deranlot, M. Basletic, A. Hamzic, A. Fert, and Y. Otani, Phys. Rev. Lett. 106, 126601 (2011).

51 L. Liu, R. A. Buhrman, and D. C. Ralph, arXiv:1111.3702 (2011).

52 K. Ando and E. Saitoh, Observation of the inverse spin hall effect in silicon (2011), arXiv:1107.2585v2.

${ }^{53}$ P. Nozières and C. Lewiner, J. Phys. (Paris) 34, 901 (1973).

54 L. L. Foldy and S. A. Wouthuysen, Phys. Rev. 78, 29 (1950).

55 W. Kohn and J. M. Luttinger, Phys. Rev. 108, 590 (1957). 
${ }^{56}$ W. K. Tse and S. Das Sarma, Phys. Rev. Lett. 96, 056601 (2006).

57 H. A. Engel, B. I. Halperin, and E. Rashba, Phys. Rev. Lett. 95, 166605 (2005).

58 J. M. Luttinger, Phys. Rev. 112, 739 (1958).

${ }^{59}$ L. Berger Phys. Rev. B 2, 4559 (1970).

${ }^{60}$ L. Berger Phys. Rev. B 5, 1862 (1972).

61 E. M. Hankiewicz, G. Vignale, and M. E. Flatté, Phys. Rev. Lett. 97, 266601 (2006).

${ }^{62}$ D. Culcer, E. M. Hankiewicz, G. Vignale, and R. Winkler, Phys. Rev. B 81, 125332 (2010).

${ }^{63}$ W. K. Tse and S. Das Sarma, Phys. Rev. B 74, 245309 (2006).

64 E. M. Hankiewicz and G. Vignale, Phys. Rev. Lett. 100, 026602 (2008).

${ }^{65}$ R. Raimondi, P. Schwab, C. Gorini, and G. Vignale, Ann. Phys. (2012).

${ }^{66}$ R. Raimondi and P. Schwab, Europhys. Lett. 87, 37008 (2009).

67 R. Winkler, Spin-orbit effects inelectron and hole systems (Springer, 2003).

68 N. A. Sinitsyn, J. Phys. Cond. Mat. 20, 023201 (2008).

69 D. Culcer and S. Das Sarma, Phys. Rev. B 83, 245441 (2011).

70 A. Mal'shukov and K. Chao, Phys. Rev. B 71, 121308 (2005).

71 O. V. Dimitrova, Phys. Rev. B 71, 245327 (2005).

72 N. Sugimoto, S. Onoda, S. Murakami, and N. Nagaosa, Phys. Rev. B 73, 113305 (2006).

${ }^{73}$ L. Hu, Z. Huang, and S. Hu, Phys. Rev. B 73, 235314 (2006).

74 J. L. Cheng and M. W. Wu, J. Phys. Cond. Mat. 20,
085209 (2008).

75 M. Borunda, T. S. Nunner, T. Lück, N. A. Sinitsyn, C. Timm, J. Wunderlich, T. Jungwirth, A. H. MacDonald, and J. Sinova, Phys. Rev. Lett. 99, 066604 (2007).

${ }^{76}$ L. D. Landau and E. M. Lifshitz, Course of Theoretical Physics, Vol. III. (Butterworth-Heinemann, Oxford, 1964).

77 Interestingly, however, the strongest spin Hall effect to date is found in inversion-symmetric metals.

${ }^{78}$ For example, a zincblende structure gives rise to Dresselhaus SO coupling, which we take as a prototypical SO coupling where needed.

79 The formalism presented in this paper applies to quasi-2D systems. No matter whether we have electrons or holes, in $2 \mathrm{D}$ they can be projected on a $2 \times 2$ subspace, though in general the two spinor components in this Hilbert space cannot be interpeted as plain "spin up" or "spin down". Instead these spinor components denote some entangled spin-orbital motion.

80 Refs. 73,74 also found a vanishing skew scattering contribution to the SHE, and similar conclusions were reached concerning the vanishing of the skew scattering contribution to the 2D anomalous Hall effect. ${ }^{75}$

81 This simplified discussion has neglected the complex relationship between the quantum well average of $\nabla V_{Q W}$ and Ehrenfest's theorem, which is covered in detail in Ref. 67.

82 This corresponds to the well-known argument that there is no skew scattering in the Born approximation ${ }^{76}$.

${ }^{83}$ For 2D hole systems and short-range impurities this term still contains the factor of 2 . In the general case of longrange scattering it is not certain that the result can be simplified in this way. 